\title{
Hilbert, Logicism, and Mathematical Existence
}

The final publication is available at Springer via http://dx.doi.org/10.1007/s11229-008-9347-1

José Ferreirós, Universidad de Sevilla

\begin{abstract}
David Hilbert's early foundational views, especially those corresponding to the $1890 \mathrm{~s}$, are analysed here. I consider strong evidence for the fact that Hilbert was a logicist at that time, following upon Dedekind's footsteps in his understanding of pure mathematics. This insight makes it possible to throw new light on the shape and evolution of Hilbert's foundational ideas, including his early contributions to the foundations of geometry and the real number system. Most interestingly, the context of Dedekind-style logicism makes it possible to offer a new analysis of the emergence of Hilbert's famous ideas on mathematical existence. And a careful scrutiny of his published and unpublished work around the turn of the century uncovers deep differences between his ideas about consistency proofs before and after 1904. Along the way, we cover topics such as the role of sets (and of the dichotomic conception of set theory) in Hilbert's early axiomatics, and detailed analyses of Hilbert's paradox and of his completeness axiom (Vollständigkeitsaxiom).
\end{abstract}

It is well known that in the address 'Axiomatisches Denken' (1918) Hilbert expressed great interest in the work of Frege and Russell, praising their "magnificent enterprise" of the axiomatization of logic, and saluting its "completion ... as the crowning achievement of the work of axiomatization as a whole" $(1918,1113)$. His high praise not only aimed at formal logic, but more particularly at logicism, as the remarkable statement that anteceded the above phrases made clear:

since the examination of consistency is a task that cannot be avoided, it appears necessary to axiomatize logic itself and to prove that number theory and set theory are only parts of logic. (Hilbert 1918, 412; emphasis mine)

This constitutes obvious endorsement of the logicistic viewpoint, but usually it is regarded as a short-lived outburst of enthusiasm, perhaps arising from study of the Principia Mathematica. ${ }^{2}$ In a summer course in 1920, Hilbert had become agnostic with regards to logicism, and in his publications of the following decade he clearly opposed that conception. Indeed, he had already expressed serious doubts in a well-known talk given in August 1904 (published as Hilbert 1905).

That this usual reconstruction is inadequate has been made clear by several unpublished documents, especially those that are coming to light thanks to the Hilbert Edition. For one thing,

\footnotetext{
Hilbert immediately went on: "However, this completion will still require new and many-sided work," which makes it clear that the "crowing achievement" was not Russell's work itself but rather its promised completion.
} 
one should pause to consider that, if we start reconstructing Hilbert's views as of 1904, we shall be missing everything he was convinced of between, roughly, 25 and 40 years old. We would ignore what might be called his "Field's medal" period! Fortunately, the edition of Hilbert's lecture courses on foundational matters is shedding a lot of new light on that period. And the evidence suggests that the 1917 address forms a unity with most of Hilbert's earlier thinking.

The development of Hilbert's foundational ideas was marked by exploration and doubts much more than his confident (but conflicting) remarks suggest. He explored in detail ideas related to all the three famous, classical foundational viewpoints - intuitionism, formalism, logicism, - and one can go as far as saying that he extracted from each of them some viewpoints and methods of lasting value. It cannot be my aim here to review all of those changes. ${ }^{3}$ I shall aim at Hilbert's early career, at logicism, since I find reason to believe that it played a much more important role for him than is usually realized.

In particular, we shall analyze the light logicism throws on the emergence of the principle that mathematical existence is nothing other than consistency. The story is intimately related with Hilbert's evolving reflections in the wake of the set-theoretic paradoxes. I shall emphasize the links between Hilbert and Dedekind, and in this connection my contribution is in line with the work of Wilfried Sieg. ${ }^{4}$ My attention was first drawn to Hilbert's early logicism by some key texts from Hilbert's lecture notes of the 1890s, quoted by Hallett in a still unpublished paper (nevertheless, Hallett did not thematize that topic). The view that Hilbert "was a logicist in Dedekind's spirit" around 1899 is also shared by Sieg \& Schlimm $(2005,157)$, but they have not entered into a detailed analysis. (As regards the influence of logicism on Hilbert's later thought, especially in the 1910s, see Mancosu 2003.)

My main claim is easy to state: the (contradictory) principle of comprehension, a central element of logic for the logicists, was the soil from which Hilbert's claim - that the consistency of an axiom system entails the mathematical existence of its objects - was to emerge. Another key point, dealt with in section 6 , is the character of consistency proofs as Hilbert envisaged

\footnotetext{
${ }_{3}^{2}$ The particular historical context, and the role of Heinrich Behman in it, has been clarified by Mancosu (2002).

See Sieg 2002, where one can find a general overview that is essentially coincident with my reconstruction.

${ }^{4}$ See especially his papers Sieg 1988 and 1990. In my book (1999), the links between Dedekind's work and Hilbert's early axiomatics were not appraised properly, one important reason being that I followed a traditional (but incorrect) way of understanding the contrast between "genetic" and "axiomatic" methods. By contrast, Sieg was the first to clearly grasp the intimate links between the methodologies of the two mathematicians; for more detailed remarks on the relations between my views and Sieg's, and clarifications about the points on which my understanding has shifted, see pp. 458-463 of the new paperback edition of Labyrinth of Thought (Birkhäuser, 2007, Epilogue).

In a recent essay (Sieg \& Schlimm 2005, 156-157), the authors have criticized my (1999) for "misjudging" the general character of Dedekind's foundational work. The claim seems to me incorrect in the main, as I have explained in pp. 461-463 of the 2007 edition, while granting the points made in the previous paragraph. This means that I agree with the points made by Sieg \& Schlimm with some important provisos in all cases. Particularly wrong seems to me the idea that the discussion on pp. 119-124 of (Ferreirós 1999) "only reveals the limitations" of our perspective; much to the contrary, I still regard that discussion, particularly what is said in pp. 122-123, as crucial for understanding the deep contrast between the freedom of axiomatic postulation in the absence of a set-theoretic background, as was the case up to 1900, or in its presence, as has been the case later.
} 
them around 1900, a topic which is likely to surprise the reader.

\section{HILBERT AND LOGICISM REVISITED.}

\subsection{Textual evidence.}

I begin by quoting a surprising statement from the introduction to the 1899 course Elemente der euklidischen Geometrie. Note that this lecture course (more precisely, this Ausarbeitung of lecture notes, due to his student $\mathrm{H}$. von Schaper) was the immediate precedent of Hilbert's legendary Grundlagen der Geometrie. And there we read:

It is of importance to establish precisely the starting point of our investigation: As given, we take the laws of pure logic and in particular all of arithmetic. (On the relationship between logic and arithmetic see Dedekind, Was sind und was sollen die Zahlen?) Our question will then be: Which propositions must we "adjoin" to the domain defined above in order to obtain Euclidean geometry? (Hilbert 2004, 303; p. 2 of the original)

First of all, this is explicit endorsement of logicism as regards arithmetic, taken in a very general sense - by "all of arithmetic" Hilbert understood the theory of the fields of real and complex numbers, and thus the basis for analysis and algebra. Dedekind $(1888,335)$ had written that "arithmetic (algebra, analysis) is only a part of logic," in reference to the "purely logical process of building up the science of numbers." Geometry, by contrast, is conceived by Hilbert as based on particular assumptions or axioms, which reflect the "facts" that concern this "natural science" (Hilbert 2004, 221; this agrees with Dedekind's understanding).

It is quite astonishing to realize that Hilbert was presenting himself as a logicist in the very same year of his pivotal contribution to geometry and axiomatization. Further documents of the years 1888, 1891, and even 1906 allow us to reinforce the point. ${ }^{6}$ In Hilbert's 1891 lecture course Projective Geometrie, one can read a passage that spells out the position behind the 1899 text:

Geometry is the theory of the properties of space. It is essentially different from the purely mathematical domains of knowledge, like, e.g., number theory, algebra, function theory. The results of these domains can be obtained by pure thought, in that one reduces the facts asserted to simpler ones through clear logical inferences, until in the end one only needs the concept of the whole number. ... Today a proposition is only then regarded as proven, when in the last instance it expresses a relationship between whole numbers. Thus, the whole number is the element. And we can obtain the concept of the whole number also by pure thought, ... The methods, the foundations of pure mathematics belong to pure thought. I need nothing else than purely logical thinking, when I occupy myself with number theory or algebra. (Hilbert 2004, 22; p. 5 of the original)

This again is a confession of logicism, with logic conceived as the science of the pure laws of thought (following a tradition that includes Boole, Dedekind, and many others). There are

\footnotetext{
${ }^{5}$ There is no correspondence to this paragraph in Hilbert's preparatory notes $(2004,221-223)$, one has to assume that these words were added in the public presentation. A natural explanation would be that the standpoint was clear in Hilbert's mind from early on, and the text of his 1891 lectures (given below; see 2004, 22) seems to confirm this explanation.

For 1906, see section 7 towards the end.
} 
reasons to think that the passage is again influenced by Dedekind, who likewise presented his views within the general context of the "arithmetisation" of pure mathematics, and defined logicism as the view that "the number concept" is an "immediate product of the pure laws of thought".

Hilbert began paying close attention to Dedekind's booklet Was sind und was sollen die Zahlen? as soon as 1888 , during his trip to Berlin as a newly established Privatdozent. He recorded that everybody in Berlin was talking about the book, but mostly in critical terms; for instance, du Bois-Reymond said that its views are "horrendous." This contrasted strongly with Hilbert's positive reaction. Even many years later, in the context of critical remarks motivated by the set-theoretic paradoxes, he always had words of high praise, saying that Dedekind's theory was "extremely sagacious" (Hilbert 1905, 130), or that it's key idea of grounding the finite upon the infinite was "dazzling and captivating" (Hilbert 1922, 162). This last must, in my opinion, be read as an autobiographical remark.

We see how the picture emerges of young Hilbert as a logicist during the 1890s, until at least 1903. In 1902, he presented geometry as "the simplest and most complete natural science" and, as in 1898, his analysis of the foundations of geometry took as given "the laws of pure logic and arithmetic [Zahlenlehre]" (2004, 540-41; p. 1 of the original). This seems to be evidence that his basic logicistic understanding of arithmetic had not changed, although I must concede that this Ausarbeitung of 1902 is more ambiguous than the previous one. Judged in this light, his outburst of enthusiasm in 1917 was nothing but a return to his earliest convictions, in the hopes that Russell's innovative type theory would, after all, prove logicism right. ${ }^{8}$

In the middle of his early logicistic period, Cantor himself informed Hilbert about the settheoretic paradoxes. The paradoxes created most serious difficulties for logicism, and Hilbert immediately set out to find remedies. His first reaction found expression in a paper dated Oct. 1899 and devoted to the number concept, Über den Zahlbegriff (Hilbert 1900). This paper, which includes the first published formulation of the idea that mathematical existence can be derived from consistency, will be at the center of the present contribution. Also at center stage will be Hilbert's second problem from the famous 1900 list presented in Paris, the problem of the consistency of arithmetic.

\subsection{Logicism in the 1890s: the influence of Dedekind.}

The first thing we need, then, is to gain a clearer understanding of what it meant for Hilbert to

\footnotetext{
${ }^{7}$ Dedekind 1888, 335-38; for another good reason to establish a link with Dedekind, see $\S 3$ below.

${ }^{8}$ The lecture course on logic given in 1917/18, on which the later Grundzüge der theoretischen Logik was based (Hilbert \& Ackermann 1928), offers further confirmation of this point. The 1928 Grundzüge offer a development of type theory in
} 
be a logicist in the 1890s. Elsewhere I have defended that one must distinguish at least two phases in the development of logicism (up to 1931): an early, triumphant phase from about 1870 to the shocking discovery of the contradictions in the years $1897-1903$, and a later phase of Russellian logicism based upon the theory of types. The 1890s was a crucial decade of explosive diffusion for this viewpoint, which found adherents in all the countries that represented mathematical powers - Dedekind, Frege, Hilbert and Schröder in Germany, Russell and Jourdain in England, Couturat in France, some of Peano's followers in Italy.

The path-breaking contributions of Frege were of very little influence until Russell and the Göttingen people (Hilbert, Zermelo) drew attention to them. In the 1890 s logicism was a foundational conception associated primarily with the name of Dedekind. This thesis comes as a surprise because it goes against historiographic usage, but one can find a great deal of evidence in its favor.

Dedekind was then a highly respected mathematician, generally regarded as one of the great masters of number theory. His work was easily available, and evidence of a very good reception abounds. Ernst Schröder adopted the logicist viewpoint in explicit reference to Dedekind; Peano's enthusiasm for the work was evidenced in his publications from 1889; even Frege's comments in 1893, albeit very critical as usual, emphasize the quality of Dedekind's work and its convergence with Frege's own. ${ }^{10}$ Last, but not least, we have seen evidence that it was precisely through Dedekind that Hilbert came to logicism.

As I have mentioned, to emphasize the role of Dedekind in the first phase of logicism is to go against historiographic tradition. Interestingly enough, this tradition comes from Bertrand Russell himself, through Russellians such as Carnap, Quine, and others. Russell was not just a prominent logician and proponent of an influential version of logicism: through his prolific writing and influence as a philosopher, he established a standard interpretation of the recent history of logic. Figures like Frege and Peano played a key role in this interpretation, while authors such as Dedekind and Schröder were sidestepped." Historians of science know well how such partisan interpretations of the past tend to be biased, and in fact they conflict with each other: Dedekind played a much more central role in Hilbert's interpretation (by which I mean his retrospective remarks, especially but not only in papers of the 1920s).

Logicism defends two key theses, the conceptual thesis (1) that the basic concepts of mathematics can be defined by logical concepts alone, and the doctrinal thesis (2) that the basic

complete adherence to Russell, which was eliminated from the second edn of 1937 (in the first, Ackermann seems to have followed uncritically Hilbert's lectures of a decade earlier).

${ }^{9}$ Ferreirós (1996) and (2001). See also my book (1999).

${ }^{10}$ See Schröder 1895, the preface and introduction to Frege 1893, and the abundant references in Peano's papers (especially his 1891) and above all in the Formulaire of 1898, which quoted Dedekind's (1888) verbatim. Dedekind's booklet saw German edns. in 1888 and 1893, the English in 1901, and a Russian one in 1905. 
principles of mathematics can be derived from logical principles. Such was exactly Dedekind's standpoint. In his view, all of arithmetic and pure mathematics can be "divest[ed] ... of [its] specifically arithmetic character" so as to be "subsumed under more general notions and under activities of the understanding without which no thinking is possible at all." And the universal (logical) principles governing such notions are such that "with [them] a foundation is provided for the reliability and completeness of proofs". ${ }^{12}$ Indeed Dedekind succeeded in reducing the whole number system to sets and mappings, to the "logical theory of sets" (Systemlehre der Logik; see Dedekind 1932, vol. 2, 112-13). Thus all of pure mathematics was proved to be an outgrowth of the pure laws of logic (modulo his conception of logic).

Most readers are puzzled to find that the concepts of set and mapping were simply regarded as logical ones. With regards to the concept of set, this was a usual view in the late $19^{\text {th }}$ century, well represented for instance with Schröder, Peano, and Russell. As Russell said from the (now remote) perspective of the early twentieth century, logic consists of three parts: the theory of propositions, the theory of classes, and the theory of relations (Russell 1903, 11) - Dedekind's logicism was based on the latter two. Both the old Aristotelian logic and the modern logical calculi were taken to deal centrally with concepts, and sets were nothing but extensions of concepts; the theory of sets or classes was simply a formal theory of concepts, a part of formal logic. For related reasons, many $19^{\text {th }}$ century authors accepted the (often unstated) comprehension principle; we shall see below that Dedekind and Hilbert did so.

With respect to the concept of mapping, Dedekind took pains (by his standards of verbal argument) to show that it was indeed indispensable to all thought, being as it is "the ability of the mind to relate things to things, to make a thing correspond to another, or to represent a thing by another" (Dedekind 1888, 335-336). This concept is "absolutely indispensable" for arithmetic and pure mathematics, as witnessed by the role of functions, isomorphisms, automorphisms, etcetera; at the same time it is sufficient to erect the building of arithmetic. Writers as influential as Schröder, Peirce, or Hilbert came to agree, although the logicians expressed a preference to reconsider Dedekind's mappings within a general theory of relations. Thus, Ernst Schröder devoted two of his Vorlesungen über die Algebra der Logik (1895) to a reelaboration of Dedekind's work on mappings and chains. Even Frege acknowledged (1893), grudgingly, the parallelism between Dedekind's system and his own on this score (with intensional concepts replacing equivalent extensional ones, as follows: set $\equiv$ concept, mapping $\equiv$

\footnotetext{
"The paradoxes also played a pivotal role, see Garciadiego (1992), Moore \& Garciadiego (1981).

Quotations from the letter to Keferstein of Febr. 1890 (Dedekind 1890), 100. By "activities of the understanding" Dedekind meant set-formation and mappings; here it seems most adequate to read his terminology in Kantian spirit, and thus not at all psychologistically. Logic is taken to be the doctrine of the "Denkgesetze," the laws of thought, but such laws govern how we should think, not the psychology of how we actually think. This distinction did not originate with Frege, but was characteristic of the Kantian treatment of logic. 
relation).

It is often said that Dedekind's work does not qualify as a reasonable form of logicism, because he did not develop a system of formal logic. Certainly, he only entered into details to the limited extent that he felt indispensable for the purpose at hand, namely, to establish a foundation for the arithmetic of natural numbers and its extension up to the complex numbers. This limited treatment meant in particular that Dedekind did not need to discuss the more elementary parts of logic having to do with the propositional connectives and the quantifiers (to be sure, he uses this logic in a clear way, but he does not theorize it). However, as I have argued at length elsewhere, it should be obvious that what is of great relevance for logicism is not the elementary layers of logical theory, but rather the higher layers. That part of logic which is essential for the logicistic project is only the upper reaches, the theory of classes and relations (resp., sets and mappings), since these are the ones that must do the work of reducing mathematical concepts and propositions.

All of the above means that, without denying the great contribution made by Frege (1879) (1893) with his analysis and formalization of elementary logic and especially quantification theory, we can regard Dedekind's contribution as a perfectly reasonable form of logicism. Indeed, a most noteworthy one, which is how Schröder, Hilbert, and others saw it.

There is additional evidence that, during the years around 1900, Hilbert relied significantly on Dedekind's foundational work. Let me add a couple of examples. Two of them will be reviewed in what follows, as both Hilbert's own set-theoretic paradox (elaborated after having been made aware of the phenomenon by Cantor) and his ideas about consistency proofs in 1900 are essentially related to Dedekind's contributions. A third example is the strong links between Dedekind's foundation of arithmetic and aspects of Hilbert's first sophisticated attempt to prove consistency (1905). Included are the axiom system employed, - a rendering of Dedekind's characterization of the structure of the natural numbers in prop. 71 of $(1888,359)$ - and some of his philosophical ideas."

\section{EXISTENCE BEFORE HILBERT.}

The modern methodology of mathematics, with its peculiarly non-constructive characteristics that pose the difficult problem of the meaning of existence claims, can be found in several $19^{\text {th }}$ century mathematicians. The main German names are Dirichlet, Riemann, Dedekind, Cantor, and Hilbert; generally speaking, mathematicians who belonged to the Göttingen tradition."

\footnotetext{
${ }^{13}$ Unfortunately, for lack of space we shall not enter into this topic in the present paper.

The new methodology emerged in the context of what I have called the "abstract conceptual approach;" see Ferreirós (1999), chap. 1. I have argued that Cantor gradually moved away from the principles of the Weierstrass school, to adopt
} 
Thus, the problem of the meaning of existence in the context of modern mathematics antedated Hilbert. Interestingly, we can find several different lines of thought that were offered to justify adoption of the modern methodology, in particular the ideas of Riemann (1868), Cantor (1883) and Dedekind. Although Cantor undoubtedly contributed to clarifying the modern methodology and showing its power with his extraordinary new ideas, one can be certain that his metaphysical excursions did little to help promote the cause. ${ }^{15}$ Hilbert was much closer to a sober logical standpoint regarding mathematics.

Some core elements in Hilbert's position remained with him throughout his life. Consider the first part of his famous conference 'On the infinite' (1926), where he discusses the role of infinity in analysis and set theory, then moves on to physics, finding that recent results suggest that "reality is finite" in all directions. Yet Hilbert argues that "it could very well be the case that the infinite has a well-justified place in our thinking and plays the role of an indispensable notion" (Hilbert 1926, 372). The problem is how to justify this intuitive idea.

Dedekind and the young Hilbert hoped to find justification in the universal laws of logic, but the older Hilbert moved on to look for metamathematical justification in his Beweisstheorie. Even though both attempts have failed, one can still share the general intuitive conviction (as this writer, for one, confesses to do).

Let us then have a closer look at Dedekind's position. In his work on the real numbers, he emphasizes (following Riemann, 1868) that, if space has a real existence, "it need not necessarily be continuous" $(1872,323)$. But even if we knew for sure that it is not continuous, nothing could prevent us from "making it continuous in thought" by the introduction of new elements. The creation Dedekind had in mind is strictly regulated by the laws of logic, as the continuous domain of the real numbers is won through "the purely logical process of building up the science of numbers" (1888, 335, see also 340-341). Thus considered, the 1872 passage contains the kernel of Dedekind's position regarding mathematical existence. The real numbers exist in a purely logical sense, not in an ontological sense: they exist "in thought," but may not correspond to physical reality. The mathematician can, by the use of pure logic, introduce continuous spaces or the set of real numbers. And he can do so in the strong sense that logic warrants such a step, including a warranty that no contradiction will emerge.

Dedekind discussed his position often in connection with Kummer's "creation" of the ideal numbers, and with his own set-theoretic elaborations upon this, the theory of ideals. This is

more and more the modern principles emanating from Göttingers like Riemann and Dedekind. The general topic of Berlin vs. Göttingen is discussed in Ferreirós (1999), 24-38; unfortunately, for reasons of space I didn't thematize the topic in later sections of the book, but chapters IV, V, VI, and VIII are full of information concerning Cantor that should be read from this viewpoint.

See the highly interesting ideas presented in Cantor (1883), $\S 8$; methodologically they are an antecedent of Hilbert's, but philosophically they display a heavy load of rationalistic and theistic metaphysics. 
obviously related with Hilbert, who would speak of the way in which the mathematician introduces "ideal elements" in the process of expanding available theories with the view to harmonize and simplify their laws or theorems, to render them more perspicuous (Hilbert 1926, 372-373). In spite of the nuances that differentiate Hilbert's views in 1926 from those he had previously held, and from Dedekind's some forty years earlier, the basic philosophical position is essentially the same. By the "creation" of mental objects, Dedekind meant a process that is always strictly regulated by logical laws. In later work, he located the essential creative step in the jump from given elements to "a new determinate [thing], their set," which is "necessarily" different from its elements (preface of 1911 to Dedekind 1888, 343).

The introduction of new mathematical objects is therefore an activity within the realm of pure thought, lacking stronger ontological implications. This position was consistent with Dedekind's logicism. Pure mathematics (arithmetic, algebra, analysis) being "only a part of logic," an immediate outflow of the pure laws of thought $(1888,335)$, the theories of pure mathematics are only limited by logical impossibility, not by any considerations of actual existence. Notice that this is not to deny that matters of mathematical existence are delicate and important: as Dedekind wrote to Lipschitz (27.07.1876), "nothing is more dangerous in mathematics than to assume existence without sufficient proof"."

I have stated that, in Dedekind's eyes, the theories of pure mathematics are only limited by logical impossibility. Reading these words conversely, the theories of pure mathematics are made acceptable by mere logical possibility (consistency). The point arose in connection with infinite sets, and this is what Dedekind had to say:

After the essential nature of the simply infinite set, whose abstract type is the number sequence $N$, had been recognized in my analysis ([1888] articles 71,73), the question arose: does such a set really exist in the realm of thought [Gedankenwelt]? Without a logical proof of existence it would always remain doubtful whether the notion of such a set might not perhaps contain internal contradictions. ((letter to Keferstein, Dedekind 1890, 101; emphases mine)

To put it in Hilbert's language, Dedekind was asking for a proof of the consistency of his axiomatisation of the theory of natural numbers; such proofs are essential to Dedekind's logicist project. Hilbert could hardly have known of Dedekind's explicit reflections as presented in the letter to Keferstein; he only knew the theoretical developments based on them, but it seems clear that Hilbert grasped the gist of his approach.

Dedekind's "logical" proofs of existence and consistency consisted in showing that there exists an infinite set as a thought-object (a Ding in the Gedankenwelt; 1888, prop. 66), and that

\footnotetext{
${ }^{16}$ Dedekind to Lipschitz, 27.07.1876, in Dedekind (1932), 477; the context is the need for a continuity axiom in order to make sure that the set of real numbers (or the corresponding domain of magnitudes) is continuous, and the contrast between Euclid's work and modern analysis. Dedekind probably had in mind, too, certain difficulties with Kummer's way of development the theory of ideal numbers (see the introduction to his French paper of 1876/77, particularly Dedekind 1932, 268).
} 
each infinite set contains a simply infinite system (1888, prop. 72). ${ }^{17}$ Notice that Hilbert endorsed the arguments in 1891 and 1899, both times implicitly. This was more obvious in 1891, as he explained that we can "obtain the concept of the whole number by pure thought, perhaps in that I can count my own thoughts" (2004, 22; p. 6 of the original). In the light of the words Hilbert had written but deleted: "in that I think something, then a second, a third, etc." (ibid. note 5), this seems to be a very brief and imprecise rehearsal of Dedekind's props. 66 and 72.

Some years later, Hilbert $(1905,131)$ would speak of the objects of mathematics as Gedankendinge, aptly putting together two of Dedekind's terms. Dedekind understood by a "thing" "any object of our thinking," indicating that sets of things are likewise thought-objects, "things" [Dinge] (Dedekind 1888, 344); and the "totality of all the things that can be an object of my thought" was called the Gedankenwelt, the "thought-world" (o.c., 358).

In assuming the mathematical existence of some "thing" (say an ideal number, or an infinite set), metaphysical considerations are completely absent, but we need a "logical proof of existence" or a consistency proof. In Dedekind's actual practice, this amounted to showing the existence of a model for the axioms, elaborated by the "purely logical" means of set theory. So, for example, "the purely logical construction of the science of numbers" helps us obtain a "continuous number-domain," the set $\mathbf{R}$ of real numbers $(1888,335)$. And "logical existence" entails consistency, since it dispels doubts as to whether, e.g., the notion of "simply infinite" set might contain internal contradictions. This was not Hilbert's formulation, but rather the converse.

Mathematical existence in the tradition of Dedekind and Hilbert is logical admissibility in the realm of pure thought. It is merely "ideal existence," as Zermelo would aptly say many years later $(1930,43)$. For Dedekind, to show that simply infinite sets exist was not to prove that there are infinitely many objects in the world - infinite sets exist in our realm of thoughts, in the Gedankenwelt. In a similar way, Hilbert proved using arithmetic that both Euclidean and nonEuclidean geometries "exist" mathematically, even though only one of these incompatible systems can be true of the physical world. ${ }^{18}$ Thus mathematical existence has a peculiar character, being far removed from the kind of existence we intend for the objects of scientific theories. We might refer to this by distinguishing purely logical existence (ideal existence) from ontological existence (real existence).

With regards to ideal or "purely logical" existence, the Hilbertian principle that consistency entails existence is quite natural... provided of course that one can envision methods of proving

\footnotetext{
${ }^{17}$ The first part is Dedekind's "theorem" of infinity (prop. 66), which has become notorious and infamous, but whose subtleties are usually bypassed (see Ferreirós 1999, 241-246).
} 
consistency that make this formulation reasonable! Initially, the natural formulation was rather that existence entails consistency. In the sequel, we shall see in more detail the way in which Hilbert's principle emerged. But first, it seems advisable to consider in more detail the role that the "logical theory of sets" had in Hilbert's actual work.

\section{ON HILBERT'S EARLY AXIOMATICS.}

In order to appreciate correctly the issues that we are dealing with, it is important to counteract the tendency of many people to view Hilbert's axiomatic work throughout his career as if it was made in one single mold. Hence the need for an excursion into his early axiomatics, underscoring the differences between this work and his later contributions in the context of proof theory. The peculiar and crucial role that sets played in Hilbert's early axiom systems will serve as a touchstone of the logicistic orientation of his early foundational work. We shall consider in some detail an important side of Hilbert's work in Grundlagen der Geometrie and in the Zahlbegriff article.

It is well known among specialists that the kind of axiomatic work produced by Hilbert at this point has to be called informal, to distinguish it from the formal axiomatizations (i.e., groups of axioms formulated within formal systems of logic) that would be developed in the 1920s in connection with the Hilbertprogramm. Informal axiomatics is still today the most frequent among mathematicians, examples being the usual axioms for groups, fields, etc. And it is crucial to realize that these early axiom systems had the theory of sets as their basis: set theory was taken to belong to the logical framework underlying the axioms. This, of course, was natural for a logicist.

All of Hilbert's early work falls within the set-theoretic tradition that led to modern mathematics. He regarded set theory as crucial for the "new mathematics" he was to promote so forcefully. His remarks in 1925 about the "Cantorian paradise" and its fruitful methods are too well-known to be quoted once more. During the golden pre-war years, in 1910, Hilbert characterized set theory as "that mathematical discipline which today occupies an outstanding role in our science, and beams out [ausströmt] its powerful influence into all branches of mathematics" (Hilbert 1935, 360). The fact that he proposed Cantor's continuum problem as the very first in his celebrated list of 1900, and the consistency problem we are discussing (which at the time, for him, was mainly a set-theoretic problem) as the second, must be viewed as part of his strong bet for the new mathematics.

Hilbert was a "man of problems" (as Blumenthal said), much more so than Dedekind, and he

\footnotetext{
${ }^{18}$ Compare the discussion in Hilbert 2004, 119-20.
} 
was very appreciative of the extremely novel questions and conjectures that Cantor was able to pose. But, as we have seen, Hilbert understood the set-theoretic methodology in the way of Dedekind, not in the Cantorian way with its strongly Platonistic, ontologico-theological underpinnings. All of his early work had strong ties with the characteristic methodology that Dedekind put forward in his researches on number theory, algebra, algebraic geometry, and foundations. Clear examples are the famous Zahlbericht (Hilbert 1897) and also Hilbert's approach in his researches on Invariantentheorie, because as Hilbert himself explained, his approach was essentially different from previous ones in that "the theory of invariants appears only as a particularly noteworthy example of the theory of fields of algebraic functions in several variables" (Hilbert 1893, 287). This emphasizes the link with the epoch-making theory of fields of algebraic functions in one variable, offered by Dedekind and H. Weber (1882). ${ }^{19}$

Less clearly realized has been the extent to which Hilbert's axiomatic work develops further the methods used by Dedekind. Despite the contributions of W. Sieg during the last 20 years, ${ }^{20}$ most historians and philosophers are still unaware of this connection and particularly of its depth. Even the Zahlbegriff paper (Hilbert 1900), with its critique of the "genetic" method in favor of the "axiomatic" one, is much closer to Dedekind's way of proceeding than may seem to a naïve reader. In Hilbert's mind the "genetic" approach was associated with Weierstrass and not Dedekind. Indeed, an examination of Hilbert's 1905 lectures on the logical principles of mathematical thought confirms this idea: in the discussion of the genetic method (Hilbert 1905a, 9-10; see also 212) he mentions only Weierstrass and Kronecker, and he accuses this method of defining things "through processes of generation, not through properties" (durch Erzeugungsprozesse, nicht durch Eigenschaften) - a reproach that can only be levelled against the Berliners, not against Dedekind. ${ }^{22}$

In Grundlagen der Geometrie, Hilbert gives axioms for the elements [Dinge] of three sets [Systeme], conventionally called points, straights and planes. The basic terminology is Dedekind's, but the important issue here is the following: Hilbert's axioms may deal with relations and operations between the elements, or just as well with conditions on sets of elements. This difference, very significant in the eyes of a modern logician, was there immaterial, because both are implicitly regarded as elementary logical methods. The same

\footnotetext{
${ }^{19}$ Dedekind and Weber established a theory of ideals for fields of algebraic curves in analogy with Dedekind's numbertheoretic ideal theory. Note that Heinrich Weber was until 1883 the only full professor of mathematics at Königsberg; Hilbert followed his lectures on elliptic functions, number theory, and a seminar on invariant theory (Hilbert 1935, 389).

${ }^{20}$ Beginning with Sieg 1988 and 1990. See in particular the recent Sieg \& Schlimm 2005.

${ }^{21}$ This should be clear after a careful study of the paper, as most of the problems that Hilbert was trying to sidestep with his axiomatic approach have nothing to do with Dedekind. Consider in particular his paragraph $(1900,184)$ about the finite character of the axiom system, as opposed to the infinitely many "possible laws" that may determine a Cantorian fundamental sequence. Only an algorithmic reading of Cantor's work, in the style of the Berlin mathematicians, can pose the problem - not the abstract one in Dedekind's style.

${ }^{22}$ On this general topic of the contraposition between Berlin and Göttingen, see Ferreirós 1999, chap. I and passim. 12
} 
happens with Hilbert's axiom system for the reals: one starts with a "system" of "things" and defines axiomatically relations and operations between them, including an unequivocally settheoretic condition, the notorious axiom of completeness (Hilbert 1900, 183; 1900a, 300). This is similar to the way in which Dedekind proceeded while characterizing structures such as number fields (1871), the ordered and complete field of the real numbers (1872), or the structure of the natural numbers (1888). In a moment we shall have a closer look at this feature.

The above may suffice to conclude that all of Hilbert's early work, from his doctoral dissertation to the turn of the century, was intimately linked with Dedekind's research fields, new concepts, and new methods. Of course, the work of Hilbert introduced many novelties in matters of content, and cannot be presented as an inessential development of what Dedekind had done earlier. That is particularly clear in his geometric work, which allowed Hilbert to explore axiom systems and their interpretations in ways that were unprecedented. But also in invariant theory and algebraic number theory, he introduced new methods and posed very advanced problems, e.g., the number-theoretic questions that would lead to class field theory. (The resonance encountered by his work is also explained by the fact that, at the time, every single new instance of success for the abstract methods was important in convincing the mathematical community, and Hilbert's results were noteworthy insofar as they made clear that all of algebra and even geometry could be approached in the "new" set-theoretic way. From a methodological angle, however, the turning point had already been reached by his predecessors.)

\subsection{Dedekind's axiomatics.}

The great difference between Hilbert and Dedekind is terminological: while the former speaks loudly and clearly of "axioms" in the modern sense, the latter refrains completely from using that word. The rationale for this option was philosophical, having to do with the fact that he still used the word in the old meaning (like Frege) and, even more importantly, with his logicistic beliefs. ${ }^{23}$ But this should not obscure the fact that the modern axiomatic methodology is clearly present in Dedekind's foundational work, and in his algebraic and number-theoretic work whether we talk of "axioms" or "conditions" [Bedingungen] should be immaterial. ${ }^{24}$

\footnotetext{
${ }^{23}$ I have elaborated on this topic in my (1999), 241-248. Concerning Frege, see his letter to Hilbert of Dec. 27, 1899 (Frege 1976, 62). Frege adhered to the "old meaning" of the word "axiom," objecting to the new meaning that "seems to emerge" in Hilbert's work, and which "I cannot quite comprehend." Incidentally, I believe it is precisely for this reason that Frege has "fundamental laws" [Grundgesetze] of arithmetic, and not at all "axioms" - once again, his views are closer to Dedekind's than is generally believed. The axiomatic style of Dedekind's work has recently been emphasized by Sieg \& Schlimm (2005), in a paper where they voice some criticisms of my book (I have answered to them in the Epilogue to the paperback edition, 2007).

Both Dedekind and Hilbert were consistent in emphasizing the arbitrary character of mathematical terminology. Their convergence on this point is noteworthy, because in all likelihood Hilbert was unaware of the relevant passages in Dedekind's correspondence (see the very noteworthy passage in an 1876 letter to Lipschitz, Dedekind 1932, 479; and also
} 
Dedekind's axiomatic style has very characteristic features. Whenever he discovers the need to introduce a new concept, he prefers to treat it separately in adequate generality; he characterizes the concept by a set of independent attributes or features (what we call axioms, following Hilbert), and explores the consequences in the corresponding degree of generality. Clear examples of this style are his treatment of the structure of module in algebraic number theory, ${ }^{25}$ and the way he treats the theory of chains in connection with set theory and arithmetic. Both examples were well-known to Hilbert. Let me take the second and expand on it.

Dedekind discovered the need for a concept of "chain" while seeking to find axioms that might characterize the number sequence (see Dedekind 1890, 99-101). This concept establishes a minimality condition, and is defined as follows:

Given a mapping $\varphi: \mathrm{S} \rightarrow \mathrm{S}$ and a subset $\mathrm{A} \subset \mathrm{S}$, the chain of $A$ is the intersection of all subsets $\mathrm{K} \subset \mathrm{S}$, such that $\mathrm{K}$ is closed under $\varphi$, and $\mathrm{A} \subset \mathrm{K}$. The chain of $\mathrm{A}$ is denoted $\mathrm{A}_{0}$ or $\varphi_{0}(\mathrm{~A}){ }^{26}$

By defining the number structure to be the chain of a singleton $\{1\}$ under an injective mapping $\varphi$ (called the successor function), the chain denoted by $\{1\}_{0}$ or $\varphi_{0}\{1\}$, Dedekind was in a position to ensure the validity of proofs by mathematical induction. But in his booklet he proceeds to study the theory of chains for arbitrary sets and arbitrary mappings (not just injective mappings); this is the topic of his section 4. And since he is dealing with the concept in this degree of generality, Dedekind $(1888,354-355)$ goes on to prove a generalized theorem of induction, formulated not for $\mathbf{N}$, but for an arbitrary chain, where again the underlying mapping is not required to be injective.

The axiomatic style of Dedekind becomes even clearer in this section of his booklet, right after the definition of the chain of set A $(1888,353$, prop. 44). From this definition he proves three simple results, namely: Prop. 45: $\mathrm{A} \subset \mathrm{A}_{0}$ (trivial); Prop. 46: $\varphi\left(\mathrm{A}_{0}\right) \subset \mathrm{A}_{0}$, which holds since $\mathrm{A}_{0}$ is closed under $\varphi$; and Prop. 47: if $\mathrm{K} \subset \mathrm{S}$ is closed under $\varphi$, and $\mathrm{A} \subset \mathrm{K}$, then $\mathrm{A}_{0} \subset \mathrm{K}$. And then he adds:

Remark: It is easy to convince oneself that the concept of the chain $\mathrm{A}_{0}$ defined in 44 is characterized completely by the previous propositions $45,46,47$.

It is impossible to work more clearly in the axiomatic style, without using the very word "axiom." The remark simply means that propositions 45, 46, 47 suffice to axiomatise the structure of chain.

the letter to Keferstein (1890), 100-01); it might be that the topic had come out in personal conversations, but at any rate the convergence underscores how close their styles of thinking were. One should note an implicit but omnipresent way in which Dedekind emphasized the arbitrariness of terminology: his striking terminological choices, e.g., "body" for fields, the meaning of "multiple" and "divisor" of two fields, or the "ideals."

${ }^{25}$ See Dedekind 1871, $§ 161,242-245$; or his 1894, $\S \S 168-172,493-524$ (corresponding to Dedekind 1932, 60-90).

Here I modernize slightly the terminology, in a way that is immaterial to the point we are discussing; compare Dedekind (1888), 353. Likewise, in the next sentence I write $\{1\}_{0}$ instead of Dedekind's ambiguous notation $1_{0}$. 14 
While Peano's axiom of induction renders directly the customary principle, Dedekind was able to subsume it under the very general and powerful theory of chains. This theory yields also the Cantor-Bernstein theorem, ${ }^{27}$ and it is not too difficult to generalize it for use in higher set theory, as Zermelo discovered. The working style of Dedekind in his axiomatic analysis of $\mathbf{N}$ is also characteristic of his later work (including algebraic number theory) in one further aspect: he had a clear tendency to prefer "set-theoretic foundations," to use Emmy Noether's apt phrase. $^{28}$

Many other features of the booklet are also relevant in connection with axiomatics. Whenever Dedekind feels the need to introduce some mathematical concept for his analysis of the natural numbers and their arithmetic, he antecedes a study of that concept in a general setting. Besides the example just given, another very relevant one is the theory of (primitive) recursive definitions in $\S 9$ of the booklet; this is subsequently applied to the basic arithmetic operations, and to the definition and theory of finite cardinals. Also relevant is the fact that, in $\S$ 10 , Dedekind $(1888,376)$ studied the models or interpretations of his axiomatic characterization of $\mathbf{N}$, proving that any two models are isomorphic. This is certainly one of the very first instances of a model-theoretic study in the mathematical literature.

\subsection{Hilbert's axiomatics in $\mathbf{1 8 9 9}$.}

Most of Hilbert's axioms for either geometry or the real numbers state conditions on the elements, and therefore can be formalized in first-order logic. In this respect, his axiomatic style was closer to Peano's and simpler than Dedekind's (who preferred to characterize and study structures directly in terms of sets, subsets, and mappings; not at the more elementary level of operations on the elements of the set). But Hilbert felt free to formulate axioms postulating conditions on sets of elements; to formalize them, one needs to quantify over sets of elements. The conspicuous example of this trait is the famous Axiom of Completeness [Vollständigkeit] that Hilbert included first in his axiomatization of the real numbers (1900), and then in subsequent editions of the Grundlagen der Geometrie.

There is a well-established tendency in the literature to say that Hilbert's completeness axiom is metamathematical, because in its formulation he would be speaking about the relation between axioms and models; indeed, Hilbert talks about a certain set that "erfüllt" (satisfies) some axioms. But this seems to be an anachronistic reading, too strongly informed by modeltheoretic ideas that would only start to crystallize from about 1915 (see Badesa 2004). Some writers present Hilbert's Axiom of Completeness as one that is second-order, but this again

\footnotetext{
${ }^{27}$ See Dedekind 1888, 356; Dedekind 1932, 447-448; and Ferreirós 1999, 239-241.
} 
cannot be true to his spirit: once more the remark is anachronistic, nobody at the time had isolated first- or second-order logic. In my view, there is a more interesting way of understanding what Hilbert did - more interesting from the philosophical point of view, and probably also more correct conceptually and historically as an interpretation of his work.

In my opinion, the situation with Hilbert's Axiom of Completeness is closely parallel to the situation with Dedekind's Chain Axiom that $\mathbf{N}=\varphi_{0}\{1\}$, i.e., that the set of natural numbers is the chain of a singleton under the successor mapping. However, Dedekind's chain condition is a minimality requirement, while Hilbert's Completeness Axiom aims to ensure maximality. And this also entails an important difference between both.

In the case of the reals, Hilbert's axiom of completeness says that they form a set of elements which is maximal for the remaining axioms (which characterize an Archimedean ordered field). His original formulation was the following:

IV 2. (Axiom of Completeness.) It is not possible to add to the set [System] of numbers another set of things, in such a way that all the axioms I, II, III, IV 1 are satisfied [erfüllt] in the set obtained by their reunion; or in short: the numbers form a set of things which is incapable of any extension while maintaining all the axioms. [Hilbert 1900, 183; also in (1900a), 300]

In a sense, Hilbert was just reasoning in ways that were becoming customary for algebra and number theory: one defines a certain kind of structure (there, number field, or ring of algebraic integers, or ideal within such a ring) and thinks about all possible realizations of the structure. One can then consider the relations of inclusion, intersection, union, between such realizations, and in particular one may consider maximality (or minimality) requirements. This is exactly the situation with fields of algebraic numbers, which Dedekind (and Hilbert) defined as algebraic extensions of $\mathbf{Q}$; the minimal set is $\mathbf{Q}$ itself (every number field $K$ is such that $\mathbf{Q} \subset K$ ), the maximal realization is the field $\mathbf{C}$ of all complex numbers (always $K \subset \mathbf{C}$ ). ${ }^{30}$

Similarly, when we require the fields to be ordered and Archimedean, the maximality condition seems enough to characterize univocally the set of real numbers, with topological completeness emerging as a by-product of maximality. This seems to have been a key realization for Hilbert at the time, especially because he was interested in having a completeness axiom that would not entail the Archimedean property; this kind of independence was important for his axiomatic work.

My proposal for formalizing the Completeness Axiom would be the following. Hilbert's

\footnotetext{
${ }^{28}$ See, and contrast, McLarty 2006. This is one of the key differences between his axiom system and that of Peano (1889), which was more elementary (Peano himself seems to have perceived it; see Ferreirós 2005, 622).

${ }^{29}$ It adds to the surprising parallelism between Dedekind and Hilbert, that the former too was led to his Chain axiom by considering ways in which the set of numbers could be augmented by an additional set of things, while keeping the remaining axioms satisfied - these "intruders" would destroy the structure (see the celebrated letter to Keferstein, Dedekind 1890, point 6).

Compare Dedekind (1871), 224: "The simplest [einfachste] field is formed by all rational numbers, the biggest [grösste] by all numbers", meaning real or complex numbers, as a previous sentence makes clear. 
word "satisfy" is perfectly avoidable, its use inessential; the conjunction of the first 17 axioms of Hilbert provides us with a set-predicate. One can simply say "whenever a set $T$ has the property defined by the axioms of groups I, ..., IV.1," or, more conveniently, one can introduce the set-predicate ' $X$ is an Archimedean ordered field,' defined by the conjunction of all the 17 axioms in groups I, II, III, plus axiom IV.1. Now, the Completeness Axiom says that the set $S$ of real numbers is such that, whenever $T$ has that property and $S \subset T$, then $S=T$. This way of presenting the matter, which seems to me closest to Hilbert's own, mimicks Dedekind's presentation, concretely his prop. 47:

$$
\begin{array}{ll}
\text { Hilbert's axiom: } & \forall T \text { (if } T \text { is an Archimedean ordered field and } S \subset T, \text { then } S=T \text { ); } \\
\text { Dedekind's 47: } & \forall K \text { (if } K \text { is closed under } \varphi \text { and } A \subset K, \text { then } A_{0} \subset K \text { ). }
\end{array}
$$

The difference in the consequents (identity in one case, inclusion in the other) clearly expresses the difference between a maximality and a minimality condition.

And this is also the source of the ambiguity inherent in Hilbert's axiom. With Dedekind's procedure, we are dealing with an intersection and all chains $K$ are included in a certain set $S$; when we wrote $\forall K$ above, we meant 'all subsets $K$ of $S$ ' - we could have written $\forall K \subset S$. The ambient space is supposed to be given and, subsequently, problems of 'existence' are relatively well defined. But when Hilbert says $\forall T$, it seems that the implicit domain can only be the universal set $\mathbf{V}$, for his sets $T$ can only be supposed to be living in $\mathbf{V}$; we should write $\forall T \subset \mathbf{V}$.

This is why, with Hilbert, one gets the feeling of some ambiguity. His axiom system seems to presuppose the universal set $\mathbf{V}$ in an essential way, as Dedekind did not. More specifically, Dedekind's proof of the existence of 'simply infinite sets' (sets isomorphic to $\mathbf{N}$ ) relies merely on some given infinite set $S$ (1888, prop. 72$){ }^{31}$ Thus, it is the case that the contradictory universal set had a very limited appearance in Dedekind's axiomatization, while the Completeness axiom of which Hilbert was so proud had the effect of 'infecting' his axiom system with the antinomical virus. Funny that he wanted to use these axioms as a basis for a consistency proof, and thereby an existence proof that would bypass the problems created by the paradoxes.

The proposed way of understanding Hilbert is simply to think that set theory belongs to the underlying logic in which the axiom system is formulated. ${ }^{32}$ As we have seen, the evidence suggests that this was just Hilbert's - and of course Dedekind's - own viewpoint in the 1890s. Hilbert's tendency is to have full set theory in the logical background. The point is that, if talk of sets and elements (systems and things) is just basic logical language, there is no essential difference between Peano-style axioms affecting the elements and Dedekind-style conditions

\footnotetext{
${ }^{31}$ Because the set is Dedekind-infinite, we have an injective mapping $\varphi: S \rightarrow S$, where the image is a proper subset of $S$.
} 
affecting sets or subsets. For whenever we have a realm of things, thinking and reasoning about sets of such things is just elementary logical reasoning.

Of course, Hilbert would later learn that in foundational work one must to be more careful, that the old logical principles had been proven faulty precisely here, and one had to develop logic and mathematics simultaneously. But it seems that it was only after Frege's reaction to the Zermelo-Russell paradox, published in 1903, that Hilbert came to doubt whether set theory really belongs to pure logic, and whether pure mathematics can be reduced to logic (Hilbert 1905). Even later, in the 1920 s, he came to use formal axiom systems, which is a completely different way of working axiomatically, one that in fact is foreign to most mathematicians. But set theory kept playing a background role in regular mathematical work, and of course informal axiomatics of the older style survived. ${ }^{33}$ This may help the reader understand the key importance that Hilbert kept ascribing to set theory in the 1920s. Set theory was, after all, the repository of a whole series of "ways of forming notions and modes of inference" that are extremely fruitful in mathematics. In a word, no one would steal from $20^{\text {th }}$ century mathematicians the freedom and power of modern methods - the set-theoretic paradise (Hilbert 1926, 375-376).

\section{PROBLEMS IN THE FOUNDATIONS OF SET THEORY.}

According to my reconstruction, Hilbert's views on "truth and existence" in mathematics emerged from a logicistic understanding of set theory in terms of the principle of comprehension. He was led to revising that contradictory principle in the light of Cantor's discovery of the antinomies of set theory; this is what triggered his noteworthy inversion of previous ideas about existence and consistency. We now consider both issues.

\subsection{The dichotomic conception.}

The typical logicistic understanding of set theory, or the "logic" of classes, can be characterized, following Gödel's perceptive remarks, as the dichotomic conception. ${ }^{34}$ Any well-defined concept $P(x)$ establishes a dichotomy of all things into those that are $P$ s and those that are non- $P$ s, i. e., a partition of $\mathbf{V}$ into the class $\{x: P(x)\}$ and its absolute complement, the class $\{x: \neg P(x)\}$. Hence the name "dichotomic conception." This standpoint is based on two key assumptions:

(i) the existence of a universal set, Dedekind's Gedankenwelt, and

(ii) the principle of comprehension as a basic law of thought, which says:

\footnotetext{
${ }_{32}^{32}$ advanced this view for the first time in Ferreirós (1999), 301-302.

${ }^{33}$ Good mathematicians made sure that their axiom systems could be adjusted within axiomatic set theory; but this was usually left out of their writings (an early example is Hausdorff 1914, but consider also van der Waerden 1930, etc.).

${ }^{34}$ See Wang 1974, 537, which obviously (compare p. 538) was a text discussed with Gödel himself. 
Given a well-defined property [an open sentence] $\Phi(x)$, there is the set $S=\{x: \Phi$

$(x)\}$.

To them, of course, one adds the principle of extensionality (Dedekind 1888, 345).

In fact it is possible to lay emphasis on either (i) or (ii), since just one of them suffices for "naïve" set theory. The path from the principle of comprehension to assumption (i) is well known: simply replace $\Phi(x)$ by a truism, such as the property ' $x=x$ '. But the converse is also true: we may begin by establishing the existence of an all-encompassing domain $\mathbf{V}$ given as a set; in order to establish the principle of comprehension, the key idea is that, since $\mathbf{V}$ is assumed to be a set, any part of it should again be a set. Hence, as a well-defined concept $P(x)$ defines a subset of $\mathbf{V}$, the set $\{x: P(x)\}$ exists.

Logicians and mathematicians from Dedekind to Quine, and some beyond, have found it most natural to assume that there ought to exist a universal realm, a set encompassing all objects and in particular all sets. ${ }^{35}$ The universal set (the set of all Dinge) was indispensable for logicism, since the whole bent of Dedekind's and Frege's viewpoints was to work out general logical principles, so general that they apply to the finite and the infinite alike, from which they hoped to establish the existence of the actual infinity of natural numbers. This can only be done provided that the universal set - which, obviously enough for a mathematician, will indeed be infinite - is given at the outset. Considering the "set" (proper class) of all possible objects of thought, Dedekind proves that this Gedankenwelt $\Sigma$ is Dedekind-infinite. To do so, he must specify a one-one mapping $\phi: \Sigma \rightarrow \Sigma$ (interestingly he chose the propositional function " $x$ can be an object of my thought"), and an element in $\Sigma$ that is not in the image $\phi(\Sigma)$ (Dedekind proposed "my own self").

Surprisingly, the comprehension principle was almost nowhere stated clearly before it was proved contradictory, in spite of its crucial role for logicism and naïve set theory. Many people tend to assume that it is identical with Frege's infamous Law V, which however is simply a principle of extensionality. ${ }^{37}$ Comprehension was implicit in Frege's symbolism, which allows

\footnotetext{
${ }^{35}$ It has taken a long and winded process to move beyond that naïve assumption, towards the idea that the set-theoretic universe is not itself a set, and indeed may be indeterminate, incompletable. See Zermelo (1930) and the insightful introduction to that text by Hallett.

The proof has been despised as "psychologistic" by Russell (1919) and others, in spite of the fact that Russell himself, like Hilbert and perhaps Frege, accepted it in his early years of logicism (Russell 1903, 357-58). But there is much evidence that Dedekind's epistemological framework should be taken to be some form of Kantianism (broadly understood), and thus alien to psychologism. The "self" in Dedekind's proof is not the psychological I, but rather some kind of transcendental subject.

Looking for an explicit formulation of Comprehension, it is better to consider Riemann's pioneering work, when he defines a "manifold" as the class whose elements are the "different instantiations" of an "antecedent general concept" (Riemann 1868, 273). In this context he brings out explicitly $(1868,273-74)$ the idea that whenever such a concept is given, there is the corresponding set or manifold. Elsewhere I have argued more at length for Dedekind's and Riemann's acceptance of this principle (Ferreirós 1996 \& 1999, 47-53, 104-106). A key example of the traces of Comprehension in Dedekind can be found in his two versions of the generalized theorem of complete induction (Dedekind 1888, props. 59 and $60)$.
} 
one to form the expression $\dot{\varepsilon} F(\varepsilon)$ - read: the extension of the concept $F$ - for any given property $F(x)$; Frege assumes that there somehow is a mapping which to every concept associates an object, but he does not present it as an explicit assumption. As regards Dedekind, there are traces of Comprehension in his publications, but it seems that he purposefully avoided relying on it as the basis of the "logical theory" of sets. I presume he doubted whether the principle was general enough: there might be sets for which one cannot define a corresponding concept (in fact, Dedekind believed that language can only represent mathematical ideas in a partial and incomplete way).

As regards Hilbert, a simple fact suggests that Comprehension was deeply imbedded in his mind around 1900: he tends to use the words "concept" and "set" as synonyms. The reader can verify this statement for herself, simply by reading the formulations employed by Hilbert in his Paris lecture (1900a, 299-301), when he talks about problem 2 of the consistency of arithmetic. Another witness is his reply to Cantor, when the sagacious explorer of the transfinite presented him with the antinomy of the class of all alephs. Hilbert protested:

The collection of all alephs can be conceived as a definite well-defined set, for certainly if any thing is given, it must always be possible to determine whether this thing is an aleph or not; and nothing more belongs to a well-defined set. ${ }^{38}$

Dedekind would not have answered differently: it is enough that the concept of an aleph be well defined, this suffices (by the unstated comprehension principle) for the corresponding set to exist. Hilbert's answer is thus a perfect example of the dichotomic conception of sets.

\subsection{Hilbert and the antinomies, 1897-1903.}

The great impact of the paradoxes of set theory around 1900 was mainly due to the wide diffusion of logicism at the time. The paradoxes cast more and more doubts on the dichotomic conception, both from the point of view of the principle of comprehension and the universal set.

None other than Hilbert was the first mathematician to mention publicly the problems in set theory. This happened in his famous address on mathematical problems (1900a, 301), but also in the previous paper 'On the number concept' (1900). The context was in both cases the same, the problem of showing the consistency of the arithmetical axioms and "therefore" the existence of the set of real numbers. Thus in the earliest of these papers we read:

To prove the consistency of the above axioms [for the real numbers] one needs only a suitable modification of familiar methods of inference. In this proof I also see the proof of the existence of the totality of real numbers, or -in the terminology of G. Cantor- the proof that the system of real numbers is a consistent (finished) set. ...

If we should wish to prove in a similar manner the existence of a totality of all powers (or of all Cantorian alephs), this attempt would fail; for in fact the totality of all powers does not exist, or in Cantor's terminology- the system of all powers is an inconsistent (unfinished) set. (Hilbert

${ }^{38}$ Hilbert's original letter is lost, but this sentence is quoted verbatim by Cantor: see Meschkowski \& Nilson (1991), 390. 
$1900,184)$

As the quotation makes clear, Hilbert was relying strongly on results communicated to him by Cantor, in the course of a correspondence from Sept. 1897 onwards. ${ }^{39}$ It is also clear that Hilbert's first communication on the matter lacked the sense of disaster that would emerge in later works of Russell (1903) and especially Frege (1903). ${ }^{40}$ For instance, in the Paris talk Hilbert repeatedly declared his conviction that one can prove the admissibility and existence of sets such as the real numbers (Hilbert 1900a, 300, 301). Indeed, the Zahlbegriff paper was his first attempt to solve the problem.

But Hilbert too had strong doubts at the beginning. These were not mentioned in his publications, only discussed privately. Bernays knew that he "temporarily thought that Kronecker had probably been right," ${ }^{, 1}$ and indeed the lecture notes of 1905 read as follows:

when I found it [Hilbert's paradox, see below], I thought in the beginning that it causes invincible problems for set theory that would lead to its failure; now I firmly believe, however, that everything essential can be kept after a revision of the foundations, as always in science up to now. (Peckhaus \& Kahle 2002, 168)

This confidence and "firm belief" is already present, and forcefully so, in the Paris talk of 1900 and the Zahlbegriff paper. I am thus inclined to think that the period of doubt may have been either during 1898, or perhaps after seeing the reaction of Frege in 1903.

Names matter. Russell (1903, 101 and 523) spoke of what we call the paradoxes as "contradictions," for they were plain contradictions given his (and Frege's) standpoint at the time, which adopted the unrestricted principle of comprehension. But the Göttingen mathematicians, under the lead of Zermelo, called them "antinomies" in an explicit allusion to Kant and to their allegedly unavoidable character. ${ }^{42}$ The word "paradox," wrote Zermelo, means merely "a statement contradicting the common opinion, it doesn't contain anything of an inner contradiction (as is the case for the paradoxes of Russell and Burali-Forti, and expressed by the term 'antinomy')."' Hilbert in his lectures of 1905 used preferentially the name "paradox," but he went on to indicate that these paradoxes are a "mathematically precise version of the Kantian antinomies" [mathem. Präcissierung Kantischer Antinomieen] (1905a, 215).

Hilbert's first acquaintance with the paradoxes was a result of his encounter with Cantor during a meeting of the GDN $\ddot{A}$ (Association of German Scientists and Physicians) in

\footnotetext{
${ }^{39}$ The relevant letters were found and published by Walter Purkert; see Purkert \& Ilgauds (1987). English translations are available in Ewald (1996), vol. 2, 926-29.

${ }^{40}$ By contrast, when Dedekind first knew of the antinomies, in the course of a visit that Felix Bernstein (then still a student of Cantor's) paid him in Easter 1897, he was led to "doubt whether human thought is fully rational" - strong words for a sober and restrained man (see Bernstein's reminiscences as quoted by E. Noether in Dedekind 1932, 449).

See Sieg 1990, 289. Bernays goes on: "Now it became his goal, one might say, to do battle with Kronecker with his own weapons of finiteness," but this remark is only valid for Hilbert's work in 1904-05.

Today we prefer to call them "paradoxes," a name that waters down their relevance and suggests that they are merely the result of a misconception, of a "naïve" approach to set theory, superseded by axiomatic set theory.

Zermelo to Nelson, December 1907; quoted in Peckhaus \& Kahle (2002).
} 
Brunswick, Sept. 1897. Their conversation was interrupted when Hilbert was just raising the question whether all infinite cardinalities are Cantorian alephs. Cantor subsequently wrote a letter saying that this was a theorem that, in his opinion, he could prove rigorously. The proof exploited the fact that the totality of all alephs is not a "finished set [fertige Menge]" or, as he would say in later correspondence, is not a "consistent set [consistente Menge]"." With this letter, a very interesting exchange on the matter was started, preceding the even more detailed correspondence with Dedekind in 1899.

In retrospect, one can locate the source of the tensions in Cantor's radical expansion of the realm of set theory, which was essentially a result of his introduction of the transfinite ordinals (Cantor 1883). It was this new context that led Cantor to the paradoxes in the first place, and he recognized that they posed inescapable difficulties for logicists such as Dedekind. This is the way in which he presented the matter to Hilbert in letters of 1897/98. The arguments were mere paradoxes for Cantor, but for Dedekind's "diametrically opposite" viewpoint they were contradictions.

As we have seen, Hilbert's first reaction was typical of a logicist, since he immediately resorted to the dichotomic conception. "The collection of all alephs can be conceived as a welldefined and concrete set," he opined, for given any thing it is always possible to determine whether it is an aleph or not; "and nothing else can be required from a well-defined set." Notice how the concept of an aleph is supposed to create a dichotomy, which in turn presupposes that the universal set is given.

Two years later, Cantor would criticize this same assumption as the crucial difference between his conception of set theory and Dedekind's. Their views were "in diametrical opposition" because he rejected Dedekind's "naïve assumption that all well-defined collections, or systems, are also «consistent systems»" . Here Cantor employs Dedekind's term "System" which Hilbert was using in his papers at the time - as a synonym of "well-defined collection," that is, one given by a definite concept. According to Cantor, Dedekind always assumed that such a definite concept is sufficient for the existence of a set - the principle of comprehension. But the well-defined collections include both sets (which Cantor intended to be his "Mengen") and what we nowadays call «proper classes». Thus Cantor intimated that Dedekind's logicistic notion of set [System] was not equivalent to his own [Menge]. He was also explicit in suggesting that the paradoxes would force Dedekind to make essential changes in his work on

\footnotetext{
${ }^{44}$ Cantor intended at the time to publish a third instalment of his paper 'Contributions to the foundations of the theory of transfinite sets', but this was delayed and never came out (see Ferreirós 1999, 290-295). His attempt to prove that all powers are alephs involved also the proof that all sets can be well-ordered.

${ }^{45}$ Cantor to Hilbert, Nov. 15, 1899 (in Purkert \& Ilgauds 1987, 154); this letter deserves to be much better known, but it is not included in Ewald's collection.
} 
the foundations of the natural numbers.

In late 1897 or 1898 , Hilbert reacted by trying to make sure whether the antinomies were a real threat for the foundations of pure mathematics. This he did by looking for a contradiction based, not on Cantor's transfinite arithmetic, but on Dedekind's arithmetic of the natural numbers. The outcome was "Hilbert's paradox" obtained simply from three principles:"

(i) existence of the set of natural numbers,

(ii) existence of the set of all internal mappings of a previously given set, $\varphi: S \rightarrow S$, and

(iii) the formation of unrestricted unions.

Hilbert considered "all sets resulting from" application of these principles "arbitrarily often [beliebig oft]" (Peckhaus \& Kahle 2002, 171). The resulting union set $U$ should be closed for internal mappings, so that it encompasses all $\varphi: U \rightarrow U$, but now a Cantorian argument by diagonalisation showed how to establish a "well-defined" mapping that cannot be in $U$. As a result of this "purely mathematical" argument, which avoided the complexities of Cantor's transfinite arithmetic, Hilbert concluded (probably in 1898) that the paradoxes are indeed a real threat.

Notice that his paradox builds upon ingredients that can be taken directly from Dedekind's work: the set $\mathbf{N}$ of natural numbers (1888, props. 71-72), the concept of internal mapping $\phi: S$ $\rightarrow S$ (prop. 36), ${ }^{48}$ and an infinitary union principle (prop. 8). The way in which Dedekind formulates the principle of union $(1888,346)$ can be accused of having exactly the degree of haziness of Hilbert's principle, since Dedekind allows to form the union of "any sets whatever" (irgendwelchen); he does not require this possibly infinite collection of sets to be itself a set. ${ }^{49}$ What was not in Dedekind is "the set of all" internal mappings: this is nothing but Cantor's Belegungsmenge (Cantor 1895, 288), introduced to define the exponentiation of cardinal numbers, and which Hilbert referred to by this same name in the lectures of 1905 . Needless to say, the diagonalisation argument is also Cantorian. ${ }^{50}$

Hilbert concluded that the basic logical principles on which set theory was based needed amendment. It was imperative to set some bounds to the "arbitrariness" that was to be allowed

\footnotetext{
${ }^{46}$ See Cantor's letters to Hilbert in (Purkert \& Ilgauds 1987; translated in Ewald 1996, vol. 2), especially that of 15.11.1899 (p. 154). See also the 1899 letters to Dedekind, where Cantor clearly states that the "collection of everything that can be thought [Inbegriff alles Denkbaren]" is not a set (Cantor 1932, 443; consider also 448). It must have been clear to Dedekind that he was making reference to his "world of thoughts" [Gedankenwelt], the universal set.

${ }^{47}$ For further details, see Peckhaus \& Kahle (2002).

${ }^{48}$ For mappings, Hilbert employs here the terminology introduced by Cantor (Selbstbelegung, see Peckhaus \& Kahle (2002), 164n, 171) instead of the original terminology of Dedekind (Selbstabbildung, or Abbildung eines Systems in sich selbst, Dedekind 1888, 351, § 4). We do not know the terminology that he may have employed back in 1898 .

The same happens already in an earlier paper of Cantor $(1880,145$; the 1895 paper does not contain such a general union principle). In the lecture notes, again, Hilbert prefers to use Cantor's 1895 terminology: "Vereinigungsmenge".

Such a fusion of ideas and methods from Dedekind and Cantor was usual in Hilbert's papers and lectures at the turn of the century. This trait differentiates Hilbert from such important experts in set theory as Felix Hausdorff (1914), and it must be taken into account in order to understand why Zermelo too went on to combine the heritages of Cantor and Dedekind.
} 
in applying principles such as union, or in the (transfinite) iteration of given operations. And it was also imperative to be more careful when applying the "fundamental principle" of comprehension: to have a well-defined concept was not enough, some further cautionary requirement was needed.

Hilbert came to accept Cantor's distinction of two kinds of "collections," and the need to revise the principle of comprehension. Some years later he offered a well-known critique of Frege, which interestingly enough can be applied verbatim to his own standpoint back in 1897 (see Hilbert 1905, 130). His conclusion was that "the conceptions and means of investigation prevalent in logic, taken in the traditional sense, ${ }^{51}$ do not measure up to the rigorous demands that set theory imposes." A major goal of the investigations into the notion of number should be to avoid and clarify these paradoxes. It seems likely that this analysis coincides with the understanding that Hilbert had reached by 1899. As we shall see, the paper 'On the concept of number' was designed precisely to answer to these new goals.

\section{CONSISTENCY AND MATHEMATICAL EXISTENCE.}

In light of the paradoxes, it was clear that the principle of comprehension needed revision. Cantor himself said it as plainly as possible: one cannot start, he emphasized in the letter to Hilbert of 15.11.1899, from the "naïve assumption" that "all well-defined collections, or systems, are also «consistent systems»" (Purkert \& Ilgauds 1987, 154). From the fact that the concept of an aleph is perfectly clear, we cannot jump to the conclusion that the set of "all" alephs is a "consistent collection." Similarly for the concept of a transfinite ordinal, since the set of "all" ordinals is antinomical, and even for Dedekind's concept of "thing" as any object of our thinking: we cannot simply assume the existence of the set of "all" things (Dedekind's Gedankenwelt), given that each ordinal and each cardinal is a mathematical thing.

Notice that in all three cases, the problem arises when one considers "all." It was Hilbert's and Cantor's intuition that there ought to be no problem with "some" or even "a great many" of the relevant "things." To mention some examples of increasing complexity, the following sets should exist: - the set of natural numbers; - Cantor's second number class (the set of all denumerable ordinals $\alpha$, i.e., $\left.\omega_{0} \leq \alpha<\omega_{1}\right)$; - the set of all alephs $\aleph_{\alpha}$ for index $\alpha$ belonging to, say, the first $\omega$ number classes of Cantor. And of course, the set of real numbers should

\footnotetext{
${ }^{51}$ In his eyes, the principle of (unrestricted) union and the principle of comprehension were merely well-established principles of "traditional logic." For the historical context that made this appraisal reasonable, see my papers (1996) and (2001). In my view, only mathematicians employing the notion of set - beginning with Riemann in 1854, - and not the traditional logicians, originated the full principle of comprehension. The principle was related to a usual assumption of logicians (Ferreirós op. cit.), but only the new mathematical context provided the incentive for formulating it in the extreme
} 
similarly be unproblematic, as also the set of all real-valued functions of a real variable $\varphi: \mathbf{R} \rightarrow$

$\mathbf{R}$; etcetera.

Although Cantor's way out was unsatisfactory, since it lacked "a precise criterion" for the distinction between "consistent" and "inconsistent systems" (Hilbert 1905, 131), his perceptive diagnosis of the problem and the details of the arguments establishing his paradoxes (and Hilbert's own) were enough to set Hilbert on a productive track. First of all, the problem had to do with the unwelcome emergence of contradictions in the area of set theory, by using principles that in any event were deemed necessary for analysis (the theory of the real numbers). Second, Hilbert believed, as a result of his previous work on foundations, that in order to find a way out it was necessary to apply the axiomatic method of geometry to all other relevant areas of mathematics, beginning with arithmetic. ${ }^{52}$ But third, when the axiomatic method is applied, the task necessarily arises "of showing the non-contradictoriness [Widerspruchslosigkeit] and the completeness of these axioms" (Hilbert 1900, 181). And the question of consistency is, in this axiomatic context, a clear one: the problem is to show that a finite system of axioms cannot lead to contradictions.

The step to invoking the axiomatic method and investigating the consistency of the axiom system provided the logical criterion that was lacking from Cantor's reflections. To be consistent or not is merely a logical property of the axiom system. The fact that is was a purely logical test must have been particularly satisfactory for Hilbert as a logicist.

It deserves to be mentioned that the main meta-axiomatic questions for Hilbert in Grundlagen der Geometrie (especially in the first edition, 1899) were questions of independence, and only to a much lesser extent - one may say as a byproduct - the question of consistency. Interestingly, independence and consistency were also the main meta-axiomatic problems for Dedekind in his Was sind und was sollen die Zahlen?, according to the 1890 letter to Keferstein. ${ }^{53}$ But consistency only came center stage with the problem of the paradoxes.

\subsection{Sets - concepts - axioms systems.}

The way in which Hilbert employed the word "concept," and interchanged it quite freely with "set" or "system," deserves special mention. On the very first page of Grundlagen der

generality that turned it into a contradictory axiom. Likewise, only mathematicians such as Cantor pushed the concept of union to the point needed for Hilbert's paradox.

${ }^{52}$ See Hilbert's Vorlesungen (2004) and Hilbert 1897/98. For the historical context of his involvement with geometry, see the work of Toepell (1986) and his contributions to the centenary edition of Grundlagen der Geometrie (Leipzig, Teubner, 1999).

Specifically, Dedekind's props. 66 and 72 establish the consistency of his axioms for the natural numbers by providing a model. But in Dedekind's publications that was left unstated in explicit terms, one had to think them through, as Hilbert may have done. Dedekind's booklet addressed also, in a very interesting way, the categoricity or monomorphy of models of the axiom system; this is the topic of $\S 10$, which studies "the class of simply infinite sets" and shows that it is a class of isomorphy. 
Geometrie, Hilbert begins with "three distinct systems [sets] of things" (points, lines, and planes); then in $\S 2$ he writes that the axioms of group I "establish a connection between the concepts indicated above, namely points, straight lines, and planes" (Hilbert 1899, emphasis mine). The comprehension principle seems to be in the background, just as it was in Hilbert's letters to Cantor of 1897. In the 1905 paper discussing Frege, quoted above, he presents the comprehension principle as "the fundamental principle that a concept (a set) is defined and immediately usable if only it is determined for every object whether the object is subsumed under the concept or not" (Hilbert 1905, 130). What I am calling attention here to is the immediate jump: "a concept (a set)."

The Frege-Hilbert correspondence abounds in further examples, and so does the text in which Hilbert posed the second problem of his Paris list (1900a, 299-301). Also in Hilbert's discussion of his own paradox, as presented in the 1905 lecture notes, he says that the principle of union makes it possible to "reunite 2 sets ... into a new conceptual unity [Begriffseinheit], a new set". And the source of the paradoxes of set theory, which are obtained "by purely logical operations," is seen to lie above all in the axiom of union, the "reunion of many concepts into one common concept [Gemeinbegriff]" (Hilbert 1905a, 195). Throughout this period, the very notion of a set is immediately linked with a the notion of a Begriffseinheit, a "conceptual unity." This in itself offers further confirmation of the thesis that Hilbert was strongly influenced by logicism at the time.

We need to mention also another association of "concept." In their correspondence, Frege accused Hilbert of employing the word "axiom" in an unclear way, one of the reasons being that he was treating it as interchangeable with "definition." From this point of view, the geometric axioms are the attributes that define the concept of "a geometry;" and the axioms for the real numbers are the characteristic properties of the number concept. ${ }^{54}$ As late as Nov. 1903 we find Hilbert speaking of the "axioms that define the concept" (Frege 1976, 80). Once again, this links very naturally with Dedekind, whose "definition" [Erklärung] no. 71 characterizes the set of natural numbers by listing four "conditions" (Dedekind 1888, 359). From Hilbert's viewpoint these are nothing but the characteristic attributes, the "axioms that define the concept" of natural number. ${ }^{5 s}$ This way of considering the matter led to the well-known idea that an axiom system is an "implicit definition."

The circle of ideas in which Hilbert is moving should now seem much clearer. An axiom system can always be regarded as the definition of a concept, and a concept is always linked -

\footnotetext{
${ }^{54}$ Notice that Hilbert's answer to the question of the "number concept" was nothing but to set up 18 axioms divided into four groups (Hilbert 1900, 181-83).
} 
via the comprehension principle - with a corresponding set. But, from the paradoxes, Hilbert learnt that it does not suffice to define a "concept" such as Euclidian space (or real number) with enough precision to be able to determine whether a given object falls under it or not. The concept of an aleph seemed definite, precise enough to establish whether a given object is or is not an aleph, but the totality of alephs was not a "mathematically existing" object. "Rather, the essential point is knowledge of the noncontradictoriness of the axioms that define the concept". ${ }^{56}$

\subsection{Consistency entails existence.}

What precedes, and especially the sentence I have just quoted from Hilbert's 1903 letter to Frege, contains already the heart of the matter. But it is necessary to unpack the consequences for an interpretation of the emergence of Hilbert's views on existence.

The old principle of comprehension can be formulated as follows:

Once a well-defined concept $\Phi(x)$ is established, there exists a set $S=\{x: \Phi(x)\}$ whose elements are all objects that fall under $\Phi(x)$.

In a modern formal version, $\Phi(x)$ being any open sentence in one free variable $x$ :

$$
\exists s \forall x(x \in s \leftrightarrow \Phi(x)) .
$$

The "concept" $\Phi$ can, in Hilbert's practice, simply be the conjunction of all the axioms in some axiom system. (Actually, the open sentence or "concept" $\Phi$ could have more than one free variable - Hilbert's geometric system is many-sorted, with three different sets of basic objects.)

Now, the amendment was simply to introduce an intermediate step: before concluding the existence of set $S$, it is necessary to prove the consistency of the conditions defining $\Phi(x)$; and the axiomatic method makes this possible. One might say that the old principle of comprehension can be summarized in the words 'definiteness entails existence,' while Hilbert's 'consistency entails existence' is just a summary of the following revised principle of comprehension:

Once a well-defined concept $\Phi(x)$ is established by means of an axiom system, the set $S=$ $\{x: \Phi(x)\}$ exists if and only if the concept $\Phi$ is consistent, i.e., iff the axiom system is consistent.

Formally, $\Phi(x)$ being any open sentence in one free variable $x$ :

$$
\exists s \forall x(x \in s \leftrightarrow \operatorname{Con}(\Phi) \wedge \Phi(x)) .
$$

Thus Hilbert wrote that, in the proof of consistency for the axioms for the real numbers, "I also see" the proof of the existence of the totality of real numbers as "a consistent (finished) set"

\footnotetext{
${ }^{55}$ Now consider, e.g., the content of $\S 13$ in the Grundlagen der Geometrie, where Hilbert offers axioms for what he will call, rather idiosyncratically, "complex number-systems" (Hilbert 1902, 23). It should be obvious that this whole passage can be called a "definition" in Dedekind's sense, and that this move would be consistent with Hilbert's linguistic usage.

Hilbert to Frege, 7.11.1903, in Frege 1976, 80 (emphasis mine).
} 
(Hilbert 1900, 184). The key gain was made possible by the careful logical control that the axiomatic method makes possible. Hilbert could proudly say: "for the final presentation and the complete logical grounding of our knowledge, the axiomatic method deserves the first rank" (Hilbert 1900, 181).

Immediately thereafter, the problem of consistency and existence was discussed again in letters to Gottlob Frege. Frege adhered to the old meaning of the word "axiom", which "is present in [Hilbert's] proposition that axioms express basic facts of the intuition" (see $G d G$ (1902), 2). He objected to the new meaning of axiom that "seems to emerge" in Hilbert's work, which however "I cannot quite comprehend" (Frege 1976, 62). In Frege's view, axioms are "propositions that are true, but cannot be proven" since their origin is in spatial intuition; and "from the truth of the axioms follows that they do not contradict each other. Therefore, that requires no further proof" (Frege 1976, 63). Such sound but old-fashioned remarks motivated Hilbert's reply:

as soon as I have established an axiom, it is given [vorhanden] and "true"; ... I was very interested to see you write precisely this sentence ["from the truth of the axioms ... each other"], because for as long as I have been thinking, writing and teaching about such things, I always say exactly the opposite: If the arbitrarily established axioms do not contradict each other with all their consequences, then they are true, then the things defined through the axioms exist. That is for me the criterion of truth and existence. (letter of Dec. 29, 1899; Frege 1976, 66)

As we have seen ( $\S 2$ towards the end), it had not been so long: with a rather restricted antecedent in 1894, the available documents indicate that Hilbert started speaking this way in 1898.

The existence of the set of real numbers is derived by the force of the principle of comprehension, but only when the essential point of the consistency of the axioms that "define the concept" has been established. This is how the famous idea that consistency entails existence was meant. Seen from a different angle, however, one might say that Hilbert's revision of the comprehension principle deprives it of its force, for, as we shall see immediately, Hilbert aimed to show that the axiom system is consistent by restricted set-theoretic means, by offering a model.

\section{CONSISTENCY PROOFS AS OF 1900.}

We now consider a most intriguing aspect of Hilbert's remarks in the Zahlbegriff paper. There he expressed the opinion that "familiar methods of inference" would suffice to prove the consistency of his axiom system for the real numbers - which I will refer to as HA (Hilbert

\footnotetext{
${ }^{57}$ In this section, I present the same conclusion that can be found in (Sieg 2002, 368-372). Sieg cautiously regarded it as "a reasoned though by no means unproblematic" reconstruction, but in my view it is likely to be correct. 
arithmetic). And the claim was restated in the Paris conference on future problems of mathematics (1900a). What could he possibly have had in mind?

This is one of those instances in which careful scrutiny of Hilbert's foundational papers reveals extraordinary changes in his approach. Anyone who may have hoped to find, behind the remarks on consistency in 1900, something akin to the proof-theoretic Hilbertprogramm of the 1920s, will be highly disappointed. (At this point, one has to bear in mind that the idea of a formal proof of consistency did not exist in the $19^{\text {th }}$ century. Hilbert introduced it around 1904, in a radical move motivated by full realization of the challenge of the set-theoretic or "logical" paradoxes. Before this, people only conceived the possibility of showing consistency by producing an actual model, exactly in the way that Dedekind did. Much evidence attests to this fact. $\left.{ }^{58}\right)$

The Paris talk adds interesting information to the above, by making slightly more explicit what kind of "familiar methods" Hilbert had in mind. He was convinced that "it must be possible to find a direct proof" of consistency for HA "by means of a careful study and suitable modification of the known methods of reasoning in the theory of irrational numbers" (1900a, 300). ${ }^{59}$ This is quite astonishing, and still mysterious. Could those methods of reasoning possibly be continued fractions? Nothing of this sort is plausible. In order to get closer to knowing what Hilbert had in mind, our best chances lie in considering his contemporary lecture notes.

Indeed, at the time Hilbert had been and was offering lectures on 'Zahlbegriff und Quadratur des Kreises' (winter 1897/89, winter 1899/1900). ${ }^{60}$ These lectures on the number concept and squaring the circle were a favorite of Hilbert's throughout this period. They revised the history of the celebrated Greek problem, all the way until its definitive negative solution by Lindemann in 1882. Hilbert himself (1893) had contributed to this topic with a simplification of the proofs of transcendence for the numbers $e$ (first proved by Hermite) and $\pi$ (Lindemann).

The 1897/98 version of the lectures added detailed treatment of the set-theoretic definitions of the real numbers: this is what Hilbert meant by adding the word 'Zahlbegriff' (exactly as in the case of the paper Hilbert 1900). In the 97/98 lectures he treats the topic according to the theories proposed in 1872 by Cantor and Dedekind. Thus, in all likelihood, what Hilbert meant by "known methods of reasoning in the theory of irrational numbers" is simply the set-theoretic methods applied by Cantor and Dedekind. These, revised in light of the paradoxes, were to be the key for the consistency proof he envisioned in 1899 and 1900.

\footnotetext{
${ }^{58}$ Frege wrote to Hilbert in Jan. 1900, saying that logical consistency of a concept can only be proven by showing an object that has all the defining properties (Frege 1976, 75). As late as 1910, Russell wrote that the absence of contradictions can never be proven, except by first showing existence; this was because it is "impossible" to effect all deductions from some given premises (review of a book by G. Mannoury in Mind 19 (1910), quoted by R. Bunn in Grattan-Guinness 1980, 234).

${ }^{59}$ Sieg $(1990,289)$ emphasizes this point in connection with remarks of Bernays.
} 
Notice that, at the time, Hilbert was still accustomed to think that set-theoretic methods are but elementary logic, an application of the "laws of thought." From this point of view, in order to show that the complicated "definition" of the concept of real number (the axiom system HA) is consistent, one would be using just the elementary logical methods of the theory of sets. However, Hilbert was cautious to say that a "suitable modification" of those methods would be required. It should be clear that he was not willing to allow full leeway in using the methods employed by Cantor and Dedekind. Taking into account his paradox and his statements of the 1890s (reviewed in $\S 1$ above), we can start to understand what he meant.

It is most likely that, even as late as 1900 , Hilbert was accepting Dedekind's definition of the natural numbers, and in particular the existence of infinite sets as a logical principle. As the reader will recall, in late 1898 , despite having admitted the problem posed by the paradoxes, he could say:

As given, we take the laws of pure logic and in particular all of arithmetic. (On the relationship between logic and arithmetic, see Dedekind, Was sind und was sollen die Zahlen?) (Hilbert 2004, 303)

This sentence was not only uttered while addressing his students, but also preserved in the authorized Ausarbeitung of Hilbert's lectures, of which 70 copies were made and distributed in 1899. The only reasonable reading is that he still accepted Dedekind's way of obtaining the concept of a natural number (the set of natural numbers) from an antecedent infinite set. And he still regarded this whole way of proceeding as purely logical.

Hilbert's "purely mathematical" paradox confirms this interpretation: in order to formulate it, he followed Dedekind in accepting the infinite set $\mathbf{N}$ as given, and also the existence of the set of all mappings $\varphi: \mathbf{N} \rightarrow \mathbf{N}$. The basic "law of thought" behind this last assumption would be that, whenever a set $S$ is given, so is the set of all mappings $\varphi: S \rightarrow S$; probably Hilbert would also have been inclined to accept the following more general principle:

(A) Whenever sets $S$ and $T$ are given, so is $\{\varphi: \varphi: S \rightarrow T\}$.

Such a principle would justify Cantor's assumption that all sequences of rational numbers are given; however, Hilbert's lectures did not present the matter in this level of detail, he offered no general justification such as the principle (A) might afford.

Hilbert's paradox shows a way in which previous set-theoretic methods needed careful study and suitable modification. When he presented the matter in 1905, he concluded:

This contradiction [Widerspruch] is by no means solved yet; but one can see that it must rest upon the fact that the operation of uniting any sets or things whatsoever [irgend welcher Menge, Dinge] into new sets or totalities is not to be allowed, even though it was always used in traditional logic, and we have been careful to apply it exclusively to the natural numbers and sets arising from them,

\footnotetext{
${ }^{60}$ See Hilbert 2004, 612-13. I thank Wilfried Sieg for making accessible to me Hilbert's unpublished lecture notes during a visit to Pittsburgh in 2004, and again in 2006 while preparing the final version of this paper. 
and thus only to purely mathematical objects. ${ }^{61}$

A new mathematical logic was needed, in which the principle of union would be modified so as to introduce some restrictions. It seems likely that Hilbert thus contributed to Zermelo's axiomatisation of set theory, in which the union axiom is restricted to an antecedently given set (or more intuitively to a family of sets, Zermelo 1908, 203). Let me insist that Cantor in 1880 and Dedekind in 1888 had not introduced such restrictions into their formulations of union principles, which thus corresponded to the principle (iii) of Hilbert (see $\S 4.2$ above).

Despite the need for such revisions and clarifications, Hilbert convinced himself that the introduction of the real numbers from the natural numbers would not be blocked. Consider, for instance, Cantor's definition of $\mathbf{R}$. Deriving the set $\mathbf{Q}$ of rational numbers poses no great difficulties, one uses "exclusively the natural numbers and sets arising from them," simple sets such as classes of ordered pairs. And now, applying principle (A) as formulated above to the given sets $\mathbf{N}$ and $\mathbf{Q}$, we obtain the set of all mappings $\varphi: \mathbf{N} \rightarrow \mathbf{Q}$. Within this, we have the subset of injective mappings, which correspond to denumerable sequences of rational numbers, and among them the Cauchy or "fundamental" sequences.

Careful scrutiny of Dedekind's derivation would have confronted Hilbert with the problem whether we can adopt some form of the Power Set principle - so that to every set $S$ there corresponds another $\wp(S)$, which is the set of all its subsets. The easiest way to argue for its adoption from Hilbert's standpoint would be to think of the subsets of $S$ as given by their characteristic mappings, $\varphi: S \rightarrow\{0,1\}$. That is to say, one derives the existence of $\wp(S)$ from principle (A), as instantiated in the case $T=\{0,1\}$. To be sure, we do not know whether Hilbert came to consider anything like this, and there is nothing of the kind in the 1905 lectures. ${ }^{62}$

To summarize, the existence of the set of real numbers was, in essence, derived by means of the (revised) principle of comprehension; this is how the famous formulation 'consistency entails existence' was meant, as emphasizing the necessary intermediate step in the application of Comprehension (see $\S 5.2$ ). But comprehension was also deprived of its force because, in order to apply this revised principle, a model of the axioms had to be established in a "purely mathematical" way - it had to be shown by restricted set-theoretic means that the system HA is realizable. The consistency proof that Hilbert had in mind, as of 1900, was still of the old kind: consistency is proven by providing a model, not by proof-theoretic argument! The principles

\footnotetext{
${ }^{61}$ Peckhaus \& Kahle (2002), 172. I have modified the translation for reasons of style and emphasis.

${ }_{62}$ Quite interestingly, we do know that Cantor considered the Power Set principle in letters to Hilbert of Oct. 1898, thinking at first that this was acceptable, but only to have second thoughts (see Meschkowski \& Nilson (1991), and my discussion in the 'Epilogue 2007' to the $2^{\text {nd }}$ edn of Ferreirós 1999). This, as far as we know, was Cantor's final word: Power Set did not seem to be an admissible principle; one has to say that this position was hardly tenable, since he had no trouble accepting all $\varphi: S \rightarrow\{0,1\}$ (see Cantor 1892). It is obvious that Zermelo - who is likely to have seen those letters disagreed, but we don't know what Hilbert thought, either in 1899 or in later years.
} 
admitted for the elaboration of the model were meant to be suitably modified set-theoretic principles, building on top of that basic ground of pure mathematics that the natural numbers constitute. ${ }^{63}$

As one can see, if this reconstruction is correct, the picture of how Hilbert's axiomatic method related to the so-called "genetic method" becomes complicated. In his own words, the axiomatic method deserves the "first rank" for the "final presentation and the complete logical grounding" of our knowledge. It enables us to gain full control of our assumptions, and to make clear that, while those assumptions may be about infinite sets, the theory is finite: a matter of finite derivation from finitely many axioms. ${ }^{64}$ Perhaps the contrast with the "genetic method," as Hilbert presents it in the introductory paragraphs to his (1900), might be saved entirely by identifying the method with Weierstrass's way of presenting the number system (see $\S 3.1$ ). But if we understand the "genetic method" to be identical with Dedekind's logicistic derivation of the number system, there is no longer a clear contrast - especially because Dedekind's methods, "suitably modified," are needed in order to solve the all-important question of the consistency of the HA axioms, and therefore the problem of the existence of the set of real numbers.

\section{LATER DEVELOPMENTS.}

It is very interesting that in Jan. 1900, Frege criticized Hilbert's ideas saying that his methods of proving consistency would in the end come down to "showing an object that has the [required] properties" (Frege 1976, 75). In Sept. 1900, Frege pressed him to indicate whether he had a different kind of method, which he doubted (op. cit., 78), but he received no answer. If my reconstruction is correct, we must conclude that Frege was right. Only some years later was Hilbert in a position to answer Frege's demand. One can even speculate that, perhaps, Frege's pointed critique helped him realize the need for a different way of proving consistency.

To judge from the available evidence, the year 1903 brought deep changes to Hilbert's reflections (compare $\S 1.1$ ). The key event must have been the publication of vol. II of Frege's Grundgesetze (1903), with its devastated appendix. Even though the Göttingers knew of Russell's antinomy since about 1900, they had not fully grasped its impact. Frege's reaction awakened them from their dogmatic slumber, and Hilbert took careful notice, too, of the fact that Dedekind himself admitted the impact of the paradoxes. ${ }^{65}$ More than twenty years later,

\footnotetext{
${ }^{63}$ My reconstruction here agrees with the "reasoned, though by no means unproblematic conjecture" suggested very briefly by Sieg in his $(2002,368)$.

Even this idea was already in Dedekind: "It appears to me all the more beautiful that man can advance to the creation of the pure continuous number-domain, without relying on any notion of measurable quantity and simply by a finite set of simple steps in thought [or inferences: durch ein endliches System einfacher Denkschritte]" (1888, 340; emphasis added).

${ }^{65}$ In 1903 Dedekind no longer regarded his foundations of arithmetic as satisfactory, refusing to give permission to republish his booklet (see 1888, 343). Hilbert 1905a, 212: In der Tat hat Dedekind das persönlich zugestanden und halt seine Begründung jetzt nicht mehr für befriedigend, er lässt daher auch sogar seine Schrift nicht mehr neu auflegen. 
Hilbert reminisced that the Zermelo-Russell paradox had "a downright catastrophic effect in the world of mathematics" $(1926,375)$. This may well have been its effect in the course of meetings and discussions at the Göttingen Mathematisches Seminar in 1903.

We know that in the session of May 12, Zermelo offered a survey of Frege's recently appeared Grundgesetze, discussing Frege's theory of real numbers in parallel with those of Dedekind and Cantor; it is natural to assume that the paradoxes were also discussed. ${ }^{66}$ Hilbert must have realized that his logicistic convictions were in need of more than slight revision, and that set theory stood in need of new foundations. The shock produced by full realization of the implications of the paradoxes was probably strong: this may have been the time when Hilbert "temporarily thought that Kronecker had probably been right". ${ }^{\circ}$ But he recovered with characteristic optimism, and it became his goal, as Bernays said, "to do battle against Kronecker with his own weapons of finiteness" (ibid.).

Hilbert is likely to have begun elaborating a novel approach in the Seminar session of Nov. 3, 1903. He lectured on the foundations of arithmetic with a view to develop clearly the axiomatic standpoint and to emphasize "the principle of contradiction [as] the pièce de résistance". ${ }^{68}$ In all likelihood, the "non-contradictoriness" of elementary arithmetic (PA) was now presented as the keystone. A letter to Frege written four days later reinforces the centrality of the logicistic context:

I see the essential gap in the traditional construction of logic to be the assumption that a concept is already there [ein Begriff bereits da sei] if one can say, for any object, whether it falls under it or not - which all logicians and mathematicians have assumed until now. This seems to me to be insufficient. Rather, the decisive point is the knowledge of the consistency of the axioms which define the concept. (Hilbert to Frege, 7.11.1903, in Frege 1976, 80)

What we know about this stage in his foundational work is the revised text of Hilbert's address at the ICM in Heidelberg, 1904, as it was published one year later (Hilbert 1905). ${ }^{69}$ This paper is noteworthy for many reasons, among which I would count the deep differences with respect to the 1920s Hilbertprogramm, differences that are too often ignored. Suffice it to say that the paper marks a deep change in Hilbert's position with regard to the existence of infinite sets. This is, as far as I know, the first paper in any language that criticizes Dedekind's "theorem" of existence for infinite sets, on the basis that it is blocked by the paradoxes $(1905,131)$.

Hilbert's new position involved a great deepening of the insight that the usual set-theoretic principles have to be questioned. Hilbert tried to employ the strategy sketched in (1900) for the real numbers, bringing it back to the more basic level of Dedekind's axiom system for the

\footnotetext{
${ }^{66}$ Jahresbericht der Deutschen Mathematiker-Vereinigung 1903, 345-46.

${ }^{67}$ Bernays as reported in Reid 1970, 173.

${ }^{68}$ Jahresbericht der Deutschen Mathematiker-Vereinigung 1903, 592: der Satz vom Widerspruch die pièce de résistance.
} 
natural numbers. Hilbert's project was, again, to give a consistency proof for the axioms, but the main purpose was to justify the assumption of an infinite set as an admissible Gedankending. The key new idea was to deploy, in the consistency proof, algorithmic strategies close in spirit to Kronecker's methodology. Hilbert showed how to put this strategy in place, but only for a very simple axiom system without Infinity. Thus the idea of a syntactic consistency proof was sketched for the first time.

The new move implied also the need to abandon or at the very least revise logicism, a position that (fortunately enough) Hilbert had never defended in print. He argued that one must develop logic and arithmetic simultaneously, and suggested that the concept of set is mathematical more than purely logical (Hilbert 1905, 131). This idea, however, was probably quite unnatural to Hilbert, as shown by the evidence given above (see e.g. § 5.1) and other pieces of evidence, e.g. from the 1917 Zurich conference. ${ }^{70}$

We began this paper with Hilbert's logicistic ideas of 1917 and the usual understanding that they were but a short-lived outburst of enthusiasm. Now we are coming to consider it plausible that, quite to the contrary, what was short-lived is the critique of logicism expressed in Hilbert's address at the ICM of 1904. A piece of evidence found by Peckhaus $(1990,116)$ gives this interpretation further support; according to the mathematician Hessenberg, who was Zermelo's friend:

Zermelo is of the opinion that Hilbert's logicism is likewise impracticable [undurchführbar; "likewise" refers to Russell's], but he thinks that Poincaré's objections are unfounded. (letter to L. Nelson, Feb. 1906, in Peckhaus 1990, 116)

Similarly to the late 1910s, Hilbert must have hoped that a reform and axiomatization of "logic itself" would make it possible to prove that arithmetic and set theory are only parts of logic" $(1918,412)$. Such hopes vanished from about 1920: the lecture courses show increasing skepticism with respect to logicism, probably under the influence of Bernays (who, among other things, knew Weyl's critique of Russell's logic); no less important was the need to rely on a concrete extralogical basis (the visualizable sign-system at the basis of finitary, "contentual" mathematics) as the soil on which to build the consistency proofs for modern mathematical

\footnotetext{
${ }^{69}$ Judging from the information given in Jahresbericht der Deutschen Mathematiker-Vereinigung (1904), p. 61, the published version is not exactly Hilbert's conference of 1904 but rather a later revision, originating at the earliest from Nov. 1904.

${ }^{70}$ Hilbert said: "The question of the consistency of the axiom system for the real numbers can also be reduced to the same question for the whole numbers; this is the merit of the theories of the irrational numbers of Weierstrass and Dedekind" (Hilbert 1918, 412). The decisive role of set theory in this procedure is not even mentioned, as if the theory of sets were transparent, merely a part of elementary logic! (when Bernays took care of editing this talk for the Gesammelte Abhandlungen vol. 3, p. 153, he felt the need to add a few words). Indeed, in that talk Hilbert regarded set theory as one of the "parts of logic" (op. cit.). 
systems."

Another question that receives new light from an appreciation of Hilbert's early adherence to logicism is his reaction to Zermelo's axiomatization of set theory. Although he always thought highly of that accomplishment and its relevance for modern mathematics, it seems that it never appeared definitive to him. This is understandable, for Zermelo did not answer to the deeper question whether, and to what extent, the set-theoretic axioms could be reduced to logic. This also helps explain Hilbert's very positive, but somewhat naïve, reaction to the Principia Mathematica of Whitehead and Russell. ${ }^{12}$ Notice that, even in Hilbert's mature presentation of logic, we find what may well be traces of logicistic hopes and beliefs, the best example being his "epsilon calculus," which was to be the basis for both the introduction of the quantifiers and the axiom of choice (see Ferreirós 2001, § 2.2).

\section{CONCLUSION.}

The context of Hilbert's early logicistic convictions was central to the process by which he came to formulate his celebrated (and debated) views on mathematical existence. The principle of comprehension seems to have been in the background. When he discovered that having a well-defined "concept" was insufficient, Hilbert emphasized "the consistency of the axioms which define the concept" as "the decisive point" (letter to Frege, Nov. 1903, quoted above). As we have seen, Hilbert was led to such views through Dedekind's work on the logicistic (settheoretic) foundations of arithmetic.

Of course, this is not to deny that the process was also influenced by his studies of geometry, which in fact was the first domain for which he stated the idea that logical consistency is the only requirement for existence in mathematics. In fact, consistency and existence of a model for the axioms of geometry were proved simultaneously on the basis of real-number arithmetic, used to provide a model of the geometric axioms (Euclidean and non-Euclidean). Hilbert's handwritten notes for the 1898/99 lecture course on the foundations of Euclidean geometry read as follows:

Usual analytic geometry.

This is possible, because the properties of the real numbers do not contradict each other, but are all compatible with each other. Thus the axioms I-V are all compatible with each other. Therefore also single axioms do not contradict each other, and hence the existence [Existenz] of nonEuclidean geometry.

\footnotetext{
${ }^{71}$ Hilbert $(1926,376)$ contrasts his position with that of Dedekind and Frege, emphasizing that Kant was right when he insisted on the need for an intuitive basis. Criticism of the logicist position is already present in the lecture course of 1920 'Probleme der mathematischen Logik', pp. 22-28 (Moore 1997, 80).

For my reasons to call it naive, see Ferreirós 1999, 329-31, 357-59. The influence of Russell's logicism, and the role of Hilbert's student Heinrich Behman during the 1910s, have recently been studied by Mancosu (2003), whose work is a most interesting complement to the present contribution.
} 
To exist means that the attributes (axioms) defining the concept do not contradict each other, i.e., that it is not possible to prove, by means of all [axioms] with the exception of one, through purely logical inferences, a proposition that contradicts the last axiom. (Hilbert 2004, 282; p. 104 of the original)

Consistency was proved only relative to the consistency of real arithmetic, but remember the phraseology in the 1898/99 lectures: "As given, we take the laws of pure logic and in particular all of arithmetic". ${ }^{73}$ This viewpoint enabled Hilbert to understand his arithmetical proofs as purely logical ones.

Early on, The first sentence of Grundlagen der Geometrie is: "Wir denken uns drei Systeme von Dingen," we consider or think of three sets of things. This is meant to pose an existential assumption. However, those things are not "out there" but only in our thoughts - they are ideal things, Gedankendinge as he would say in 1905.

Starting in 1898, the paradoxes taught Hilbert that one had to be very cautious with assumptions of existence, even if we are only talking of ideal existence. The Cantorian paradoxes led him to expand the use of the axiomatic method to arithmetic, and finally to logic itself. They forced him to deepen the foundational application of his existence principle, looking for a consistency proof for the axiom system HA for the real numbers. Hilbert tended to emphasize that, in giving an axiomatic system, we assume the existence of "things" (or "systems" of things) such as described by the axioms; with the consistency proof, the existential assumption established at the outset (existence of $\mathbf{R}$ as a set) would be completely justified.

Such a way of thinking must have appeared satisfactory to a logicist, for the essential criterion that decides the whole issue is a purely logical condition ("non-contradictoriness" of the number concept $=$ the axiom system). It was also a natural reply, for, as we are talking of ideal thought-existence (Gedankendinge), it should be enough to show that its assumption is logically possible. Consistency guarantees logical possibility, and this seems entirely sufficient for ideal existence. ${ }^{74}$ On the other hand, Hilbert's idea of logic was, at this point, still the settheoretic one that we find in Dedekind. Working on this basis, the early consistency proofs literally establish the "existence" of a model satisfying the axioms - they are semantic, modeltheoretic.

The necessity to treat logical principles carefully had been clear to Hilbert right from his first acquaintance with the antinomies, 1897/98, but at the time he only looked for "suitable modifications" (indeed slight ones) of the set-theoretic principles employed by Dedekind and

\footnotetext{
${ }^{73}$ Hilbert 2004, 303; emphasis mine. We have seen essentially the same viewpoint even much later (Hilbert 1918, 412).

Hilbert's notes for a course given at Königsberg in 1894, 'Die Grundlagen der Geometrie,' present already the idea of proving consistency for hyperbolic geometry, but here he talked of "possibility" instead of "existence": "The possibility [Möglichkeit] of such [a geometry], i. e., its inner consistency [Widerspruchslosigkeit], can rather be proven strictly. For it is possible to establish (and even to define it purely arithmetically by numbers) a system of units - points, lines, planes - in which all our axioms ... are satisfied" (Hilbert 2004, 119-20; p. 87-88 in the original) 
Cantor. The situation changed rather dramatically after 1903, when the very basis of set theory, the infinitary standpoint (the axiom of infinity), was questioned. In 1903/04 Hilbert searched for refined means by which consistency could be established, and this took him to a completely new and unprecedented development, the idea of syntactic consistency proofs (see § 7).

Along the way of reviewing these developments, we have encountered what may well be more interesting findings. Particularly noteworthy seems to me the shift in Hilbert's ideas about consistency proofs, from the 1890s to 1905 . Take the assumption of the "three sets of things" with which Grundlagen begins; Hilbert justified it quite directly by showing that one can give arithmetic models: triples of real numbers for points, etc. This justification was based on building arithmetical models, and the existence of the real numbers was presupposed; ${ }^{75}$ the consistency proof was, at the very same time, a proof of existence. The surprising finding is that Hilbert's ideas about the consistency of real-number arithmetic in 1900 were no different! The situation changed completely after the idea of a syntactic proof of consistency emerged in 1904, as we saw in section $7 .^{76}$

In the 1920s Hilbert did not insist again on the idea that logical consistency of an axiom system entails the existence of its object domain. The main purpose of the celebrated Hilbertprogramm was to prove the consistency of formalized mathematical theories, period. He did not insist on the more speculative idea that this yields some kind of existence - but he did not disavow the idea either. Now Hilbert tended to emphasize the disparity between "contentual mathematics," the finitary part of the discipline constituting its most indubitable subject matter, and the "ideal elements" that are added in the course of expansion of the discipline (Hilbert 1926). But, even though this marks a stronger attitude of doubt concerning higher mathematics, the position is still coherent with the old view that mathematical existence is only some form of "ideal," quasi-logical existence.

Acknowledgements. A number of people helped me make more precise and complete my views on matters discussed here, particularly Wilfried Sieg, Michael Hallett, Ignasi Jané, and also Jeremy Gray, David E. Rowe, Volker Peckhaus, José F. Ruiz, and two referees. The topic was presented at a meeting in Seville (Sept. 2003), at the Philosophy Colloquium of Carnegie Mellon University, Pittsburgh (Apr. 2004), and at the Berkeley Working Group in History \& Philosophy of Logic, Mathematics and Science (Sept. 2006). My research has been funded by the Spanish Ministry (research project BFF2003-09579$\mathrm{C} 03)$.

\footnotetext{
${ }_{75}^{75}$ Hilbert was very explicit about this in 1902, see (2004), 563-64; see also the 1898/99 text on p. 391.

This had the side effect that the existence principle now sounded much more speculative, which may have been the ultimate cause why Hilbert ended up de-emphasizing it.
} 


\section{References.}

Badesa, C. 2004. The birth of model theory: Löwenheim's theorem in the frame of the theory of relatives, Princeton University Press, Princeton, NJ, 2004.

Bernays, P. 1935. Hilberts Untersuchungen über die Grundlagen der Arithmetik, in Hilbert, Gesammelte Abhandlungen, vol. 3 (Berlin, Springer, 1935), 196-216.

1935a. Sur le platonisme dans les mathématiques, L'Enseignement Mathématique 34, 52-69. German trans. in Bernays (1976), English in Benacerraf \& Putnam (1983), 258-71.

1976. Abhandlungen zur Philosophie der Mathematik, Darmstadt, Wissenschaftliche Buchgesellschaft.

Cantor, G. 1883. Grundlagen einer allgemeinen Mannichfaltigkeitslehre. Ein mathematischphilosophischer Versuch in der Lehre des Unendlichen. Leipzig: Teubner. Also (without preface) in Mathematische Annalen 21, and in Cantor (1932), 165-208; references to the latter. English trans. in Ewald (1996), vol. 2.

1892. Über eine elementare Frage der Mannigfaltigkeitslehre, Jahresbericht der DMV 1, 75-78. References to Cantor (1932), 278-280. English trans. in Ewald (1996), vol. 2.

1895. Beiträge zur Begründung der transfiniten Mengenlehre, MA 46, 481-512. References to Cantor (1932), 282-311.

1932. Gesammelte Abhandlungen mathematischen und philosophischen Inhalts, ed. E. Zermelo, Berlin, Springer. Reprint Hildesheim, G. Olms, 1966.

Dedekind, R. 1871. Über die Komposition der binären quadratischen Formen, Supplement X to 2nd edn. of J.P.G. Lejeune-Dirichlet, Vorlesungen über Zahlentheorie, ed. Dedekind, 4th edn., Braunschweig, Vieweg, 1871. References to the partial reprint in Gesammelte Werke, vol. 3 (Vieweg, 1932), 223261.

1872. Stetigkeit und irrationale Zahlen, reprinted in Gesammelte Werke, vol. 3 (Braunschweig, Vieweg, 1932). English translation in Ewald (1996), vol. 2.

1888. Was sind und was sollen die Zahlen?, reprinted in Gesammelte Werke, vol. 3 (Braunschweig, Vieweg, 1932). English translation in Ewald (1996), vol. 2.

- 1890. Letter to Keferstein, 27.02.1890, English translation in Van Heijenoort (1967), 98-103. First published in H. Wang, The axiomatisation of arithmetic, Journal of Symb. Logic 22 (1957), 145-158.

Dedekind, R. \& H. Weber. 1882. Theorie der algebraischen Funktionen einer Veränderlichen, Journal für die reine und angewandte Mathematik 92 (1882) (dated Oct. 1880). In Dedekind's Gesammelte Werke, vol. 1, (Braunschweig, Vieweg, 1930), 238-350.

Ewald, W. 1996. From Kant to Hilbert: A source book in the foundations of mathematics, vol. 2, Oxford University Press.

Ferreirós, J. 1996. Traditional Logic and the Early History of Sets, 1854-1908. Archive for History of Exact Sciences 50: 5-71.

1997. Notes on Types, Sets and Logicism, 1930-1950. Theoria (San Sebastián) 12, 91-124.

1999. Labyrinth of Thought. A history of set theory and its role in modern mathematics. Basel/ Boston: Birkhäuser. 
- 2001. The Road to Modern Logic - An interpretation, The Bulletin of Symbolic Logic 7, 441-484.

2005. R. Dedekind (1888) \& G. Peano (1889), in I. Grattan-Guinness (ed.), Landmark Writings in Western Mathematics 1640-1940, Elsevier, Amsterdam, 2005, chapter 47, pp. 613-626.

Frege, G. 1879. Begriffschrift, Halle, Nebert. Reprint in Hildesheim, Olms, 1964.

— 1893. Grundgesetze der Arithmetik, vol. 1. Jena, Pohl. Reprint Hildesheim, Olms, 1966.

1903. Grundgesetze der Arithmetik, vol. 2. Jena, Pohl. Reprint Hildesheim, Olms, 1966.

— 1976. Wissenschaflicher Briefwechsel, Hamburg, Felix Meiner.

Garciadiego, A. 1992. Bertrand Russell y los orígenes de las "paradojas" de la teoría de conjuntos.

Madrid, Alianza Editorial. English version in Basel, Birkhäuser, 1992.

Grattan-Guinness, I. (ed.) 1980. From the Calculus to Set Theory, 1630-1910, London, Duckworth.

Gray, J. J. 2000. The Hilbert Challenge, Oxford University Press.

Hallett, M. (to appear). Hilbert on Number, Geometry and Continuity. Forthcoming in The Bulletin of Symbolic Logic.

Van Heijenoort, J. 1967. From Frege to Gödel. A source book in mathematical logic, 1879-1931, Cambridge/London, Harvard Univesity Press.

Hilbert, D. 1893. Über die vollen Invariantensysteme, Mathematische Annalen 42, 313-373. Reprint in Gesammelte Abhandlungen, Berlin, Springer, vol. 2, 287-344.

1897. Bericht über die Theorie der algebraischen Zahlen, Jahresbericht der DMV 4. Reprint in Gesammelte Abhandlungen, Berlin, Springer, vol. 1, 1932. English translation as The Theory of Algebraic Number Fields, Berlin / New York, Springer, 1998.

— 1897/98. Zahlbegriff und Quadratur des Kreises (Vorlesungen, Winter-Semester 1897/98), Universitäts-Bibliothek Göttingen, Cod. Ms. 549. Publication forthcoming in Hilbert's Lectures on the Foundations of Arithmetic and Logic, 1894-1917 (eds. W. Ewald, M. Hallett, W. Sieg \& U. Majer), Berlin, Springer.

1899. Grundlagen der Geometrie, in Festschrift zur Feier der Enthüllung des Gauss-Weber Denkmals in Göttingen. Leipzig, Teubner. $7^{\text {th }}$ edn, revised and with additions, Berlin, Springer, 1930. Centenary edn, Leipzig, Teubner, 1999.

- 1900. Über den Zahlbegriff, Jahresbericht der Deutschen Mathematiker-Vereinigung 8, 180-184. Reprinted with some changes in the $7^{\text {th }}$ edn. of Hilbert (1899), but references are to the original. English translation in Ewald (1996), vol. 2.

_ 1900a. Mathematische Probleme, Nachrichten von der königlichen Gesellschaft der Wissenschaften zu Göttingen, 1900, p. 253-297. References to the reprint in Gesammelte Abhandlungen, vol. 3, Berlin, Springer, 1935, 290-329. English translation in Gray (2000).

— 1905. Über die Grundlagen der Logik und Arithmetik, Verhandlungen des dritten internationalen Mathematiker-Kongresses in Heidelberg, Leipzig: Teubner, 174-185. References to the English translation in van Heijenoort (1967), 129-138.

1905a. Logische Principien des mathematischen Denkens (Vorlesungen, SS 1905, ausgearbeitet von

E. Hellinger), Mathematisches Institut der Universität Göttingen. To appear in vol. 2 of David Hilbert's Lectures on the Foundations of Mathematics and Physics (Berlin, Springer). 
1918. Axiomatisches Denken, Mathematische Annalen 78, 405-15. Reprinted in Gesammelte Abhandlungen, vol. 3, Berlin, Springer, 1935, 146-56. References to the original edn.

1922. Neubegründung der Mathematik, Abhandlungen mathematischen Seminar Universität Hamburg 1, 157-77. References to the reprint in Gesammelte Abhandlungen, vol. 3, Berlin, Springer, 1935, 157-77. English trans. in [Ewald 1996], vol. 2.

1926. Über das Unendliche, Math. Annalen 95, 161-90. Shortened version in [Hilbert 1930]. References to the English translation in [van Heijenoort 1967], 367-92

— 2004. David Hilbert's Lectures on the Foundations of Geometry, 1891-1902, eds. M. Hallett \& U. Majer, Berlin, Springer, 2004.

Hilbert, D. \& W. Ackermann, 1928. Grundzüge der theoretischen Logik, Berlin, Springer.

Mancosu, P. 1998. From Hilbert to Brouwer: The debate on the foundations of mathematics in the 1920s, Oxford Univ. Press.

- 2003. The Russellian Influence on Hilbert and his School, Synthese 137, pp. 59-101.

McLarty, C. 2006. Emmy Noether's “Set-theoretic” Topology: From Dedekind to the rise of functors, in J. Ferreirós \& J. Gray, eds., The Architecture of Modern Mathematics, Oxford University Press, 2006.

Meschkowski, H. \& W. Nilson. 1991. Georg Cantor: Briefe, Berlin, Springer.

Moore, G. H. 1997. Hilbert and the Emergence of Modern Mathematical Logic. Theoria 12 (1997), 65-90.

Moore, G. H. \& A. Garciadiego 1981. Burali-Forti's Paradox: A reappraisal of its origins, Historia Mathematica 8, 319-50.

Peano, G. 1889. Arithmetices principia, nova methodo exposita, Torino, Bocca. Partial English trans. in [van Heijenoort 1967], 83-97.

- 1891. Sul concetto di numero, Rivista di matematica, 1, 87-102, 256-267. Also in Opere scelte, vol. 3, Rome: Cremonese, 1959, 80-109.

— 1898. Formulaire de mathématiques, vol. II, art. 2: Arithmétique, Turin : Bocca, 1898. Extract in Opere scelte, vol. 3, Rome: Cremonese, 1959, 215-231 (see also 232-248).

Peckhaus, V. 1990. Hilbertprogramm und kritische Philosophie, Göttingen, Vandenhoeck \& Ruprecht.

Peckhaus, V. \& R. Kahle 2002. Hilbert's Paradox. Historia Mathematica 29 (2002), no. 2, 157-175.

Purkert, W. \& H. J. Ilgauds 1987. Georg Cantor 1845-1918, Base1/Boston/Stuttgart, Birkhäuser.

Reid, C. 1970. Hilbert, New York/Berlin, Springer.

Riemann, B. 1868. Über die Hypothesen, welche der Geometrie zu Grunde liegen [1854], in Gesammelte mathematische Werke (Berlin, Springer/Teubner, 1990), 272-287. Translation in Ewald (1996), vol. 2.

Rowe, D. E. 2000. The Calm Before the Storm: Hilbert's early views on foundations, in V. F. Hendricks et al. (eds.) Proof Theory, Kluwer Acad. Pub., 2000, pp. 55-93.

Russell, B. 1903. The principles of mathematics, Cambridge University Press. $2^{\text {nd }}$ edn. 1937. Reprint London, Allen \& Unwin, 1948.

1919. Introduction to Mathematical Philosophy, London, Allen \& Unwin.

Schröder, E. 1895. Vorlesungen über die Algebra der Logik, vol. 3, Leipzig: Teubner. Reprint: New York, Chelsea, 1966. 
Sieg, W. 1988. Hilbert's programs: 1917-1922, The Bulletin of Symbolic Logic 5, 1-44.

_ 1990. Relative Consistency and Accessible Domains, Synthese 84, 1990, 259-297. Reprinted in J. Ferreirós \& J. Gray, eds., The Architecture of Modern Mathematics, Oxford University Press, 2006.

2002. Beyond Hilbert's Reach?, in D. Malament (ed.), Reading Natural Philosophy - Essays in the History and Philosophy of Science and Mathematics (Open Court Press), 363-405.

Sieg, W. \& D. Schlimm. 2005. Dedekind's Analysis of Number: Systems and Axioms, Synthese 147, 121-170.

Toepell, M. 1986. Über die Entstehung von David Hilberts “Grundlagen der Geometrie”, Göttingen, Vandenhoeck \& Ruprecht.

Weyl, H. 1918. Das Kontinuum: Kritische Untersuchungen über die Grundlagen der Analysis, Leipzig, Veit. References to the reprint New York, Chelsea.

1946. Mathematics and Logic. A brief survey serving as a preface to a review of «The Philosophy of Bertrand Russell», American Mathematical Monthly 53 (1946), 2-13.

Zermelo, E. 1908. Untersuchungen über die Grundlagen der Mengenlehre, I, Math. Annalen 65, 261-281. References to English translation in [van Heijenoort 1967], 199-215.

1930. Über Grenzzahlen und Mengenbereiche, Fundamenta Mathematicae 16, 29-47. English translation in Ewald (1996), vol. 2. 